\title{
Global well-posedness and long-time behavior of the fractional NLS
}

\author{
Mouhamadou $S y^{1}\left[\mathbb{D} \cdot\right.$ Xueying $\mathrm{Yu}^{2}$
}

Received: 19 December 2020 / Revised: 4 July 2021 / Accepted: 9 August 2021 /

Published online: 30 August 2021

(c) The Author(s) 2021

\begin{abstract}
In this paper, our discussion mainly focuses on equations with energy supercritical nonlinearities. We establish probabilistic global well-posedness (GWP) results for the cubic Schrödinger equation with any fractional power of the Laplacian in all dimensions. We consider both low and high regularities in the radial setting, in dimension $\geq 2$. In the high regularity result, an Inviscid - Infinite dimensional (IID) limit is employed while in the low regularity global well-posedness result, we make use of the Skorokhod representation theorem. The IID limit is presented in details as an independent approach that applies to a wide range of Hamiltonian PDEs. Moreover we discuss the adaptation to the periodic settings, in any dimension, for smooth regularities.
\end{abstract}

Keywords Fractional NLS · Compact manifold · Invariant measure · Almost sure global well-posedness

Mathematics Subject Classification 35A01 · 35Q55 - 35R11 $\cdot 60 \mathrm{H} 15 \cdot 37 \mathrm{~K} 06 \cdot 37 \mathrm{~L} 50$

The first author's (M.S.) current address is Department of Mathematics, Imperial College London, United Kingdom. This work was written at Department of Mathematics, University of Virginia, Charlottesville, VA

The second author's (X.Y.) current address is Department of Mathematics, University of Washington, Seattle, WA. This work was written at Department of Mathematics, MIT, Cambridge, MA.

Mouhamadou Sy

m.sy@imperial.ac.uk

Xueying Yu

xueyingy@uw.edu

1 Department of Mathematics, Imperial College London, Huxley Building, London SW7 2AZ, UK

2 Department of Mathematics, University of Washington, C138 Padelford Hall Box 354350, Seattle, WA 98195, USA 


\section{Contents}

1 Introduction . . . . . . . . . . . . . . . . . . . . . . . . . . 1262

1.1 Motivation . . . . . . . . . . . . . . . . . . . . . . . . . . . . . . . 1263

1.2 History and related works . . . . . . . . . . . . . . . . . . . . . . . . . . . . . . . 1264

1.3 Invariant measure as a tool of globalization . . . . . . . . . . . . . . . . . . . 1264

1.4 Main results . . . . . . . . . . . . . . . . . . . . . . . . . . . . 1265

1.5 Adaptation to the periodic case . . . . . . . . . . . . . . . . . . 1268

1.6 Comparison with $[52,54] \ldots \ldots$. . . . . . . . . . . . . . . . . . . . . . . 1269

1.7 Organization of the paper . . . . . . . . . . . . . . . . . . . . . . . . . . 1270

2 Preliminaries . . . . . . . . . . . . . . . . . . . . . . . . 1270

2.1 Notation . . . . . . . . . . . . . . . . . . . . . . . . . . . . . . . 1270

2.2 Eigenfunctions and eigenvalues of the radial Dirichlet Laplacian on the ball . . . . . . . 1270

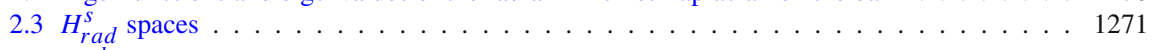

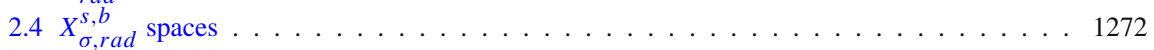

2.5 Useful inequalities . . . . . . . . . . . . . . . . . . . . . . . . . . . . 1273

3 Description of the Inviscid-Infinite dimensional (IID) limit . . . . . . . . . . . . . . . 1275

3.1 Abstract version of Bourgain's invariant measure argument . . . . . . . . . . . . . 1275

3.1 .1 Individual bounds . . . . . . . . . . . . . . . . . . . . . . . . . . . . . . 1277

3.1 .2 Statistical ensemble . . . . . . . . . . . . . . . . . . . . . . . . . . . . . . . 1279

3.1 .3 Globalization . . . . . . . . . . . . . . . . . . . . . . . . . . . 1280

3.1 .4 Invariance results . . . . . . . . . . . . . . . . . . . . . . . . . 1281

3.2 Construction of the required invariant measures . . . . . . . . . . . . . 1283

4 Proof of Theorem 1.1: Probabilistic part . . . . . . . . . . . . . . . . . . . . 1286

4.1 Fulfillment of Assumption 5 (The dissipation inequality and time of local existence) . . . . 1287

4.2 Fulfillment of Assumption 6 (The stochastic GWP) . . . . . . . . . . . . . . . . . . 1289

4.3 Fulfillment of Assumption 7 (Zero viscosity limit, $\alpha \rightarrow 0$ ) . . . . . . . . . . . . . 1291

4.4 Estimates . . . . . . . . . . . . . . . . . . . . . . . . . . . . . . . . . . 1294

5 End of the proof of Theorem 1.1: Local well-posedness _ . . . . . . . . . . . . . . . . . . 1297

5.1 Bilinear Strichartz estimates . . . . . . . . . . . . . . . . . . . . . . . . . . . . . . 1298

5.2 Nonlinear estimates . . . . . . . . . . . . . . . . . . . . . . . . . . . . . . . 1301

5.3 Local well-posedness . . . . . . . . . . . . . . . . . . . . . . . . . . . 1305

6 Proof of Theorem $1.3 \ldots \ldots \ldots$. . . . . . . . . . . . . . . . . . . . 1308

6.1 Existence of global solutions and invariance of the measure . . . . . . . . . . . 1308

6.2 Uniqueness and Continuity . . . . . . . . . . . . . . . . . . . . . . 1310

APPENDIX A. Results on probability measures and stochastic processes . . . . . . . . . . . . 1312

A.1. Convergence of measures . . . . . . . . . . . . . . . . . . . . . . . . . . 1312

A.2. Stochastic processes . . . . . . . . . . . . . . . . . . . . . . . . . 1313

APPENDIX B. Some important lemmas . . . . . . . . . . . . . . . . . . . . . . . 1314

References . . . . . . . . . . . . . . . . . . . . . . . . . . . . . 1315

\section{Introduction}

In this work, we consider the initial value problem of the cubic fractional nonlinear Schrödinger equation (FNLS)

$$
\partial_{t} u=-\mathbf{i}\left((-\Delta)^{\sigma} u+|u|^{2} u\right),\left.\quad u\right|_{t=0}=u_{0}
$$

on both the $d$-dimensional torus $\mathbb{T}^{d}, d \geq 1$, and the unit ball $B^{d}, d \geq 2$, supplemented with a radial assumption and a Dirichlet boundary condition

$$
\left.u\right|_{\partial B^{d}}=0 .
$$


Here $\sigma \in(0,1]$. When $\sigma=1$ we have the standard Schrödinger equation. The FNLS equation satisfies the following conservation laws referred as the mass and the energy

$$
\begin{aligned}
M(u) & =\frac{1}{2}\|u\|_{L^{2}}^{2}, \\
E(u) & =\frac{1}{2}\|u\|_{\dot{H}^{\sigma}}^{2}+\frac{1}{4}\|u\|_{L^{4}}^{4} .
\end{aligned}
$$

It also enjoys the scaling invariance given by

$$
v=\lambda^{\frac{\sigma}{2}} u\left(\lambda^{\frac{1}{2}} x, \lambda^{\sigma} t\right)
$$

Consequently, its critical regularity is given by the Sobolev space $H^{s_{c}}$ where

$$
s_{c}=\frac{d}{2}-\sigma .
$$

Data of regularity weaker than $s_{c}$ (resp. stronger than $s_{c}$ ) are called supercritical (resp. subcritical). The equation is called energy-supercritical (resp. energy-criticical, energy-subcritical) if $s_{c}>\sigma$ (resp. $s_{c}=\sigma, s_{c}<\sigma$ ); that is, $\sigma<\frac{d}{4}$ (resp. $\sigma=\frac{d}{4}$, $\left.\sigma>\frac{d}{4}\right)$. Since $\sigma \in(0,1)$, the cubic FNLS equations have energy-supercritical ranges in all dimensions $d \leq 3$, and is fully energy-supercritical in dimensions $d \geq 4$. In this paper we address all the scenarios listed above. We construct global solutions by mean of an invariant measure argument and establish long-time dynamics properties.

\subsection{Motivation}

In recent decades, there has been of great interest in using fractional Laplacians to model physical phenomena. The fractional quantum mechanics was introduced by Laskin [42] as a generalization of the standard quantum mechanics. This generalization operates on the Feynman path integral formulation by replacing the Brownian motion with a general Levy flight. As a consequence, one obtains fractional versions of the fundamental Schrödinger equation. That means the Laplace operator $(-\Delta)$ arising from the Gaussian kernel used in the standard theory is replaced by its fractional powers $(-\Delta)^{\sigma}$, where $0<\sigma<1$, as such operators naturally generate Levy flights. Also, it turns out that the equation (1.1) and its discrete versions are relevant in molecular biology as they have been proposed to describe the charge transport between base pairs in the DNA molecule where typical long range interactions occur [46]. The continuum limit for discrete FNLS was studied rigorously first in [36]. See also [27,28,33] for recent works on the continuum limits.

The FNLS equation does not enjoy the strong dispersion estimates as the classical NLS does. The bounded domain setting naturally highlights this lack of dispersion. This makes both the well-posedness and the long-time behavior more difficult than the Euclidean setting on one hand, an the classical NLS on the other hand.

The problem of understanding long-time behavior of dispersive equations on bounded domains is widely open. While on Euclidean spaces scattering turns out 
to apply to most of the defocusing contexts, on bounded domains one does not expect such scattering and there is no well-established general asymptotic theory. It is however expected that solutions would generically exhibit weak turbulence ${ }^{1}$. See for example $[7,12,16,29,31,32,41,49]$ and references therein for results in the direction of weak turbulence. A way to detect a weak turbulent behavior is to analyze the higher order Sobolev norms of the solutions and determine whether or not the quantity

$$
\limsup _{|t| \rightarrow \infty}\|u(t)\|_{H^{s}}
$$

is infinite for some $s$.

In this paper, we prove probabilistic global well-posedness for (1.1) by mean of invariant measures and discuss the long-time behavior by employing ergodic theorems. In particular, we show that a slightly different version of (1.2) remains bounded almost surely with respect to non-trivial invariant measures of FNLS.

\subsection{History and related works}

Let us briefly present some works related to (1.1). The authors of [21] employed a high-low method, originally due to Bourgain [8], to prove global well-posedness for (1.1) posed on the circle with $\sigma \in\left(\frac{1}{2}, 1\right)$ below the energy space. Local wellposedness on subcritical and critical regularities and global existence for small data was proved in [23], the authors of [23] established also global well-posedness in the energy space for some powers $\sigma$ depending on the dimension, see also [30] where a convergence from the fractional Ginzburg-Landau to the FNLS was obtained. FNLS with Hartree type nonlinearity was studied in [13]. On the circle, Gibbs measures were constructed in [50] and the dynamics on full measure sets with respect to these measures was studied. The authors of the present paper proved the deterministic global well-posedness below the energy space for FNLS posed on the unit disc by extending the I-method (introduced by Colliander, Keel, Staffilani, Takaoka and Tao [15]) to the fractional context [55].

\subsection{Invariant measure as a tool of globalization}

Bourgain [5] performed an ingenious argument based on the invariance of the Gibbs measure of the nonlinear one-dimensional NLS to prove global existence on a rich set of rough data. The Gibbs measure of the one-dimensional NLS is supported on the space $H^{\frac{1}{2}-}$ and was constructed by Lebowitz, Rose and Speer [44]. The non existence of a conservation law at this level of regularity was a serious issue for the globalization. The method introduced by Bourgain to solve this issue was to derive individual bounds from the the statistical control given by the Fernique theorem. A crucial fact is that these bounds do not depend on the dimension of the Galerkin projections of the equation which are used in the construction of the Gibbs measure.

\footnotetext{
$1_{1}$ Here weak turbulence refers to an energy cascade from low to high frequencies as time evolves. The norms higher than the energy tend to blow up at infinity.
} 
With such estimates, the local solutions of the equation are globalized by an iteration argument based on comparison with the global solutions of the finite-dimensional projections. This is how an invariant measure can play the role of a conservation law. More precisely, we should say how the role played by a conservation law survive through the invariant measure; indeed such quantity are essentially constructed by the use of a conservation laws and can be seen as their statistical duals. This approach is widely exploited in different contexts. For instance, we refer the reader to the papers $[6,9,11,22,56,57]$ and references therein.

A second approach based on a fluctuation-dissipation method was introduced by Kuksin [38] in the context of the two-dimensional Euler equation, and developed in the context of the cubic NLS by Kuksin and Shirikyan [39]. This method uses a compactness argument and relies on stochastic analysis with an inviscid limit. The Hamiltonian equation is viewed as a limit as viscosity goes to 0 of an appropriately scaled fluctuation-dissipation equation that enjoys a stationary measure for any given vicosity. By compactness, this family of stationary measures admit at least one accumulation point as the viscosity vanishes, this turns out to be an invariant measure for the limiting equation. The scaling of the fluctuations with respect to the dissipation is such that one obtains estimates not depending on the viscosity. These estimates allow to perform a globalization argument. See $[25,40,51,53]$ for works related to this method.

The two approaches discussed above were developed on various setting. However for energy supercritical equations, both approaches come across serious obstructions. The first author initiated a new approach that combines the two methods and applied it to the energy-supercritical NLS [52], this approach was developed further by the authors in [54]. This approach utilizes a fluctuation-dissipation argument on the Galerkin approximations of the equation, we then have a double approximation: a finite-dimensional one and a viscous one. Two limits are considered in the following order: (i) an inviscid limit to recover the Galerkin projection of the Hamiltonian equation, (ii) then an infinite-dimensional limit to recover the equation itself. In the infinite-dimensional limit, a Bourgain type iteration is used. Overall, different difficulties arise in adapting the Bourgain strategy to a non Gaussian situation. That is why new ingredients were involved. This method is what we refer to Inviscid-Infinitedimensional limit, or simply the "IID" limit. See also the work [43] for a similar procedure. We perform in Sect. 3 a general and independent version of the IID limit.

\subsection{Main results}

We set the regularity index for local well-posedness

$$
s_{l}(\sigma)= \begin{cases}\frac{d}{2}, & \text { if } \sigma \in\left(0, \frac{1}{2}\right), \\ \frac{d-1}{2}, & \text { if } \sigma \in\left[\frac{1}{2}, 1\right), \\ \frac{d-2}{2}, & \text { if } \sigma=1 .\end{cases}
$$

Once we fix the dimension and the fractional powere in the FNLS equaation, that is $d \geq 1$ and $\sigma \in(0,1]$, we define the following interval $I_{g}(d, \sigma)$, which is the range of 
globalization in Theorem 1.1.

$$
I_{g}:=I_{g}(d, \sigma)=\cup_{i=1}^{3} I_{g}^{i}(d, \sigma),
$$

where $I_{g}^{i}:=I_{g}^{i}(d, \sigma), i=1,2,3$ are defined as follows

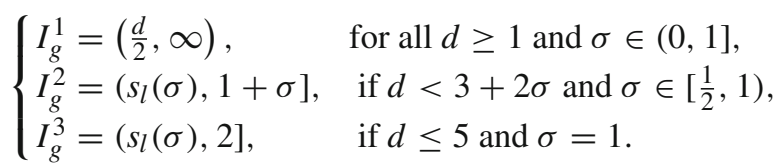

See Remark 1.5 below for a comment on the intervals $I_{g}$; it is a result of constraints imposed by the local well-posedness index above and statistical estimations on the dissipation of the energy in Sect. 4.

Theorem 1.1 Let $\sigma \in(0,1]$ and $d \geq 2$. Let $s \in I_{g}$, and $\xi: \mathbb{R} \rightarrow \mathbb{R}$ be a one-toone concave function. Then there is a probability measure $\mu=\mu_{\sigma, s, \xi, d}$ and a set $\Sigma=\Sigma_{\sigma, s, \xi, d} \subset H_{\text {rad }}^{s}\left(B^{d}\right)$ such that

(1) $\mu(\Sigma)=1$;

(2) The cubic FNLS is globally well-posed on $\Sigma$;

(3) The induced flow $\phi_{t}$ leaves the measure $\mu$ invariant;

(4) We have that

$$
\int_{L^{2}}\|u\|_{H^{s}}^{2} \mu(d u)<\infty
$$

(5) We have the bound

$$
\left\|\phi_{t} u_{0}\right\|_{H^{s-}} \leq C\left(u_{0}\right) \xi(1+\ln (1+|t|)) \quad t \in \mathbb{R}
$$

(6) The set $\Sigma$ contains data of large size, namely for all $K>0, \mu\left(\left\{\|u\|_{H^{s}}>K\right\}\right)>0$.

Remark 1.2 Notice the strong bound obtained in (1.6). This is to be compared with the bounds obtained in the Gibbs measures context which are of type $\sqrt{\ln (1+t)}$ (see for instance $[5,56,56,57])$.

Theorem 1.1 is based on a deterministic local well-posedness. However, for $\sigma \leq \frac{1}{2}$ or $s \leq \frac{d-1}{2}$, we do not have such local well-posedness. We have a different result in this case. Set the probabilistic GWP interval for Theorem 1.3 below:

$$
J_{g}=J_{g}^{1} \cup J_{g}^{2}
$$

where

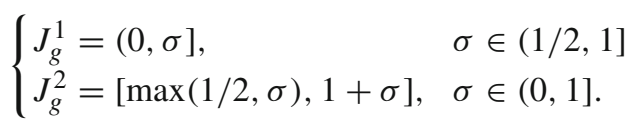


Theorem 1.3 Consider $\sigma \in(0,1]$ and $s \in J_{g}$, let $d \geq 2$. There is a measure $\mu=$ $\mu_{\sigma, s, d}$ and a set $\Sigma=\Sigma_{\sigma, s, d} \subset H_{r a d}^{s}\left(B^{d}\right)$ such that

(1) $\mu(\Sigma)=1$;

(2) The cubic FNLS is globally well-posed on $\Sigma$;

(3) The induced flow $\phi_{t}$ leaves the measure $\mu$ invariant;

(4) We have that

$$
\int_{L^{2}}\|u\|_{H^{s}}^{2} \mu(d u)<\infty
$$

(5) The set $\Sigma$ contains data of large size, namely for all $K>0, \mu\left(\left\{\|u\|_{H^{s}}>K\right\}\right)>0$.

A common corollary to Theorems 1.1 and 1.3 is as follows

Corollary 1.4 For any $u_{0} \in \Sigma$, where $\Sigma$ is any of the sets constructed in Theorems 1.1 and 1.3, there is a sequence of times $t_{k} \uparrow \infty$ such that

$$
\lim _{k \rightarrow \infty}\left\|u_{0}-\phi_{t_{k}} u_{0}\right\|_{H^{s}}=0
$$

The corollary above is a direct application of the Poincare recurrence theorem and describes a valuable asymptotic property of the flow. Another corollary of interest is a consequence of the Birkhof ergodic theorem [37] ${ }^{2}$. From (1.5) and (1.7), we have that for the data $u_{0}$ constructed in Theorems 1.1 and 1.3,

$$
\limsup _{T \rightarrow \infty} \frac{1}{T} \int_{0}^{T}\left\|\phi_{t} u_{0}\right\|_{H^{s}}^{2} d t<\infty .
$$

The quantity above is slightly weaker than that given in (1.2). Even though (1.8) does not rule out weak turbulence for the concerned solutions, it gives to a certain extent an 'upper bound' on the eventual energy cascade mechanism. The estimate (1.8) is especially important in the context of the data constructed in Theorem 1.1 where the regularity $H^{s}$ can be taken arbitrarily high.

Remark 1.5 The intervals given in (1.4) can be explained as follows: the result of Theorem 1.1 requires a deterministic local well-posedness and a strong statistical estimate. This statistical estimate is obtained by using the dissipation operator $\mathscr{L}(u)$ defined in (4.4). The operator $\mathscr{L}(u)$ is defined differently on low and high regularities. In fact the estimates in low regularities $\max \left(\sigma, \frac{1}{2}\right) \leq s \leq 1+\sigma$ rely on a use of a Córdoba-Córdoba inequality (see Corollary 2.5 ) while the high regularity estimates $s>\frac{d}{2}$ use an algebra property. It is clear that the FNLS has a good local well-posedness on $H^{s}$ for $s>d / 2$ for all $\sigma \in(0,1]$. This explains the globalization interval given in the first scenario in (1.4). However, in low regularities the local well-posedness (LWP)

\footnotetext{
2 Notice that we use here the version of the Birkhof ergodic theorem (Theorem 2.3 in [37]) that doesn't require ergodicity of the dynamics under the considered measure. Therefore, we then loose in the characterisation of the limits of the orbital averages. However the claim does not need such characterisation, it requires only the finiteness of the limits which holds true with the invariance property of the measure.
} 
is only valid for some indexes: (i) for $\sigma=1$, LWP holds for $s>\frac{d-2}{2}$, then we can globalize for $\max \left(\sigma, \frac{1}{2}, \frac{d-2}{2}\right)=\max \left(1, \frac{d-2}{2}\right)<s \leq 1+\sigma$ which necessitates $d \leq 5$; hence the third scenario in (1.4). (ii) For $\sigma \in\left[\frac{1}{2}, 1\right)$, LWP holds for $s>\frac{d-1}{2}$, which leads to the second scenario in (1.4).

Let us also remark that for the classical cubic NLS $(\sigma=1)$, an invariant measure was constructed in dimension $d \leq 4$ on the Sobolev space $H^{2}$ in [39]. See also [52] for higher dimensions and higher regularities invariant measures for the periodic classical $\operatorname{NLS}(\sigma=1)$.

The complete description of the supports of fluctuation-dissipation measures is a very difficult open question. Only few results are known on substantially simple cases compared to nonlinear PDEs (see e.g. $[3,45]$ ). In the context of nonlinear PDEs, it is traditional to ask about qualitative properties to exclude some trivial scenarios. Without giving details of computation we refer to [48] and Theorem 9.2 and Corollary 9.3 in [52] as a justification of the following statement which is valid for the different settings presented in Theorems 1.1 and 1.3.

Theorem 1.6 The distributions via $\mu$ of the functionals $M(u)$ and $E(u)$ have densities with respect to the Lebesgue measure on $\mathbb{R}$.

Remark 1.7 The equation (1.1) admits planar waves as solutions, we cannot exclude the scenario that the measures constructed here are concentrated on the set of these trivial solutions. However, this property is very unlikely because of the scaling between the dissipation and the fluctuations which leaves a balance in the stochastic equation (4.5): If the inviscid measures were concentrated on planar waves, the latter should be very close to the "complicated" solutions of (4.5) (these are not trivial at all because of the noise and the scaling); for small $\alpha$ and large $N$, this would result in a non-trivial and highly surprising attractivity of the planar waves in the dynamics of (1.1).

\subsection{Adaptation to the periodic case}

Theorem 1.8 (1) Let $d \geq 1, \sigma \in(0,1]$ and $s>\frac{d}{2}$, the result of Theorem 1.1 are valid on $\mathbb{T}^{d}$.

(2) The result of Theorem 1.3 are valid on $\mathbb{T}^{d}$ for:

(a) $d=1,2$ and $s \in[\max (1 / 2, \sigma), 1]$ for all $\sigma \in(0,1]$;

(b) $d=3$ and $s \in[\max (1 / 2, \sigma), 1]$ for all $\sigma \in(1 / 2,1]$;

Remark 1.9 (1) For the periodic extension of Theorem 1.1 given in item (1.8), we just notice that the smoothness of the regularities $(s>d / 2)$ allows a naive fixed point argument (without using Strichartz estimates) for a local well-posedness result and the calculation in Sect. 4 can be used to obtain the exponential bounded needed in the Bourgain argument.

(2) The argument behind the extensions in item (1.8) above is essentially the fact that the uniqueness argument in Theorem 1.3 is based on the radial Sobolev inequality which requires an $H^{\frac{1}{2}+}$ regularity. This regularity however is not enough for the periodic case if $d \geq 2$. Nevertheless, for $d=1,2$ and $\frac{d}{2}<s \leq 1+\sigma$, we 
have uniqueness in $H^{s}\left(\mathbb{T}^{d}\right)$, thanks to the embedding $H^{s} \subset L^{\infty}$, and the same procedure gives the claim for $d=3$. However, once $d \geq 4$, Theorem 1.3 fails on $\mathbb{T}^{d}$ because $\frac{d}{2} \geq 1+\sigma$ for all $\sigma \in(0,1]$.

\subsection{Comparison with $[52,54]$}

The probabilistic technique employed in this paper is closed to [52,54]. It is worth mentioning the novelty in the present work beside the fact that fractional NLS equations are much less understood than the standard NLS which motivated our interest to the problem considered here, and the presentation of the IID limit in a general form that is independent of characteristics of (1.1). A main difference from [52,54] is the fact that the result of Theorem 1.3 goes below the energy space, and $s$ can go all the way toward 0 . The major issue in achieving this is the uniqueness in low regularity. Our strategy is to control the gradient of the nonlinearity, that is the term $|u|^{2}$, and then to combine it with the radial Sobolev embedding and an approximation argument. This control is anticipated in the preparation of the dissipation operator.

Let us present the dissipation operator (see (4.4)) on which the low regularity result (see e.g. Sect. 6) is based, in particular:

$$
\mathscr{L}(u)=e^{\xi^{-1}\left(\|u\|_{H^{s-}}\right)}\left(\Pi^{N}|u|^{2} u+(-\Delta)^{s-\sigma} u\right) \quad 0<s \leq 1+\sigma, \quad \sigma \in(0,1] .
$$

The dissipation rate $\mathscr{E}(u)$ of the energy will be of regularity $s$ which, for $s<\sigma$, is weaker than the energy. Since $\mathscr{E}(u)$ is the highest regular quantity controlled, we are then in new ranges of regularity compared to [52,54]. To deal with these ranges we need new inputs at different levels of the proof. For instance, the large data argument relies on the identity (4.17) whose proof cannot be achieved by using the approach of [52] and [54] (where the dissipation was of positive order and then the dissipation rate of the energy was smoother than the energy itself which made it useful in the derivation of the identity concerning the dissipation rate of the mass). We, instead, introduce a modified approach which use a careful cutoff on the frequencies.

It is worth mentioning the central quantity that we manage to control at the finitedimensional level (the control is however uniform in the dimension) by the use of a fluctuation-dissipation strategy:

$$
e^{\xi^{-1}\left(\|u\|_{H^{s-}}\right)}\left(\left\||u|^{2}\right\|_{\dot{H}^{\sigma}}^{2}+\left\|\Pi^{N}|u|^{2} u\right\|_{L^{2}}^{2}+\|u\|_{H^{s}}^{2}+\left\||u|^{2}\right\|_{\dot{H}^{s-\sigma}}^{2}\right) .
$$

Below is the role of each term in the quantity above:

- The term $\|u\|_{H^{s}}$ determines the minimal regularity of the measure

- The term $\left\||u|^{2}\right\|_{\dot{H}^{\sigma}}^{2}+\left\||u|^{2}\right\|_{\dot{H}^{s-\sigma}}^{2}$ combined with the radial Sobolev inequality and an approximation argument allows to obtain uniqueness of solutions in Theorem 1.3 (see Sect. 6.1). To this end we need either $\sigma>\frac{1}{2}$ or $s-\sigma>\frac{1}{2}$, this results in a control of gradient of the nonlinearity $|u|^{2} u$ far from the origin of the ball, and then an approximation argument introduce in our previous work [54] is employed. 
- The term $\left\|\Pi^{N}|u|^{2} u\right\|_{L^{2}}^{2}$ combined with the Skorokhod representation theorem and a compactness argument allows to pass to the limit $N \rightarrow \infty$ and prove the existence of solutions.

- The term $e^{\xi^{-1}\left(\|u\|_{H^{s-}}\right)}$ is employed in the Bourgain argument to obtain, in particular, the concave bounds on the solutions claimed in Theorem 1.1.

\subsection{Organization of the paper}

We present the inviscid - infinite dimensional (IID) limit in details in Sect. 3. We consider a general Hamiltonian PDE and present the general framework of the IID limit, formulate assumptions and, based on them, prove the essential steps of the method. Section 4 is devoted to fulfill the assumptions made in Sect. 3. Section 5 ends the fulfillment of the assumptions by establishing the local well-posedness one, hence the proof of Theorem 1.1 is complete. Section 6 is devoted to the proof of Theorem 1.3.

\section{Preliminaries}

In this section we present some notations, functions spaces, properties the radial Laplacian and useful inequalities.

\subsection{Notation}

We define

$$
\|f\|_{L_{t}^{q} L_{x}^{r}(I \times D)}:=\left[\int_{I}\left(\int_{D}|f(t, x)|^{r} d x\right)^{\frac{q}{r}} d t\right]^{\frac{1}{q}},
$$

where $I$ is a time interval and $D$ is either the ball $B^{d}$ or the torus $\mathbb{T}^{d}$.

For $x \in \mathbb{R}$, we set $\langle x\rangle=\left(1+|x|^{2}\right)^{\frac{1}{2}}$. We adopt the usual notation that $A \lesssim B$ or $B \gtrsim A$ to denote an estimate of the form $A \leq C B$, for some constant $0<C<\infty$ depending only on the a priori fixed constants of the problem. We write $A \sim B$ when both $A \lesssim B$ and $B \lesssim A$.

For a real number $a$, we set $a-$ (resp. $a+$ ) to represent to numbers $a-\epsilon$ (resp. $a+\epsilon)$ with $\epsilon$ small enough.

For a metric space $X$, we denote by $\mathfrak{p}(X)$ the set of probability measures on $X$ and $C_{b}(X)$ is the space of bounded continuous functions $f: X \rightarrow \mathbb{R}$. If $X$ is a normed space, $B_{R}(X)$ represents the ball $\left\{u \in X \mid\|u\|_{X} \leq R\right\}$.

\subsection{Eigenfunctions and eigenvalues of the radial Dirichlet Laplacian on the ball}

From Sect. 2 in [2], one has the following bound for the eigenfunctions of the radial Laplacian 


$$
\left\|e_{n}\right\|_{L_{x}^{p}\left(B^{d}\right)} \lesssim \begin{cases}1, & \text { if } 2 \leq p<\frac{2 d}{d-1}, \\ \ln (2+n)^{\frac{d-1}{2 d}} & \text { if } p=\frac{2 d}{d-1} \\ n^{\frac{d-1}{2}-\frac{d}{p}}, & \text { if } p>\frac{2 d}{d-1} .\end{cases}
$$

We have also the asymptotics for the eigenvalues

$$
z_{n} \sim n
$$

\section{$2.3 H_{r a d}^{s}$ spaces}

Recall that $\left(e_{n}\right)_{n=1}^{\infty}$ form an orthonormal bases of the Hilbert space of $L^{2}$ radial functions on $B^{d}$. That is,

$$
\int e_{n}^{2} d L=1
$$

where $d L$ is the normalized Lebesgue measure on $B^{d}$. Therefore, we have the expansion formula for a function $u \in L^{2}\left(B^{d}\right)$,

$$
u=\sum_{n=1}^{\infty}\left\langle u, e_{n}\right\rangle e_{n}
$$

For $s \in \mathbb{R}$, we define the Sobolev space $H_{r a d}^{s}\left(B^{d}\right)$ on the closed unit ball $B^{d}$ as

$$
H_{\text {rad }}^{s}\left(B^{d}\right):=\left\{u=\sum_{n=1}^{\infty} c_{n} e_{n}, c_{n} \in \mathbb{C}:\|u\|_{H^{s}\left(B^{d}\right)}^{2}=\sum_{n=1}^{\infty} z_{n}^{2 s}\left|c_{n}\right|^{2}<\infty\right\} .
$$

We can equip $H_{\text {rad }}^{s}\left(B^{d}\right)$ with the natural complex Hilbert space structure. In particular, if $s=0$, we denote $H_{\text {rad }}^{0}\left(B^{d}\right)$ by $L_{\text {rad }}^{2}\left(B^{d}\right)$. For $\gamma \in \mathbb{R}$, we define the map $\sqrt{-\Delta}^{\gamma}$ acting as isometry from $H_{\text {rad }}^{s}\left(B^{d}\right)$ and $H_{\text {rad }}^{s-\gamma}\left(B^{d}\right)$ by

$$
\sqrt{-\Delta}^{\gamma}\left(\sum_{n=1}^{\infty} c_{n} e_{n}\right)=\sum_{n=1}^{\infty} z_{n}^{\gamma} c_{n} e_{n}
$$

We denote $S_{\sigma}(t)=e^{-\mathbf{i} t(-\Delta)^{\sigma}}$ the flow of the linear Schrödinger equation with Dirichlet boundary conditions on the unit ball $B^{d}$, and it can be written as

$$
S_{\sigma}(t)\left(\sum_{n=1}^{\infty} c_{n} e_{n}\right)=\sum_{n=1}^{\infty} e^{-\mathbf{i} t z_{n}^{2 \sigma}} c_{n} e_{n}
$$




\section{$2.4 X_{\sigma, r a d}^{s, b}$ spaces}

Using again the $L^{2}$ orthonormal basis of eigenfunctions $\left\{e_{n}\right\}_{n=1}^{\infty}$ with their eigenvalues $z_{n}^{2}$ on $B^{d}$, we define the $X^{s, b}$ spaces of functions on $\mathbb{R} \times B^{d}$ which are radial with respect to the second argument.

Definition 2.1 ( $X_{\sigma, \text { rad }}^{s, b}$ spaces $)$ For $s \geq 0$ and $b \in \mathbb{R}$,

$$
X_{\sigma, r a d}^{s, b}\left(\mathbb{R} \times B^{d}\right)=\left\{u \in \mathscr{S}^{\prime}\left(\mathbb{R}, L^{2}\left(B^{d}\right)\right):\|u\|_{X_{\sigma, \text { rad }}^{s, b}\left(\mathbb{R} \times B^{d}\right)}<\infty\right\}
$$

where

$$
\|u\|_{X_{\sigma, \text { rad }}^{s, b}\left(\mathbb{R} \times B^{d}\right)}^{2}=\sum_{n=1}^{\infty}\left\|\left\langle\tau+z_{n}^{2 \sigma}\right\rangle^{b}\left\langle z_{n}\right\rangle^{s} \widehat{c_{n}}(\tau)\right\|_{L^{2}\left(\mathbb{R}_{\tau}\right)}^{2}
$$

and

$$
u(t)=\sum_{n=1}^{\infty} c_{n}(t) e_{n}
$$

Moreover, for $u \in X_{\sigma, \text { rad }}^{0, \infty}\left(\mathbb{R} \times B^{d}\right)=\cap_{b \in \mathbb{R}} X_{\sigma, \text { rad }}^{0, b}\left(\mathbb{R} \times B^{d}\right)$ we define, for $s \leq 0$ and $b \in \mathbb{R}$, the norm $\|u\|_{X_{\sigma, r a d}^{s, b}\left(\mathbb{R} \times B^{d}\right)}$ by (2.3).

Equivalently, we can write the norm (2.3) in the definition above into

$$
\|u\|_{X_{\sigma, r a d}^{s, b}\left(\mathbb{R} \times B^{d}\right)}=\left\|S_{\sigma}(-t) u\right\|_{H_{t}^{b} H_{x}^{s}\left(\mathbb{R} \times B^{d}\right)} .
$$

For $T>0$, we define the restriction spaces $X_{\sigma, T}^{s, b}\left(B^{d}\right)$ equipped with the natural norm

$$
\|u\|_{X_{\sigma, T}^{s, b}\left(B^{d}\right)}=\inf \left\{\|\tilde{u}\|_{X_{\sigma, r a d}^{s, b}\left(\mathbb{R} \times B^{d}\right)}:\left.\tilde{u}\right|_{(-T, T) \times B^{d}}=u\right\} .
$$

Lemma 2.2 (Basic properties of $X_{\sigma, r a d}^{s, b}$ spaces)

(1) We have the trivial nesting

$$
X_{\sigma, \text { rad }}^{s, b} \subset X_{\sigma, \text { rad }}^{s^{\prime}, b^{\prime}}
$$

whenever $s^{\prime} \leq s$ and $b^{\prime} \leq b$, and

$$
X_{\sigma, T}^{s, b} \subset X_{\sigma, T^{\prime}}^{s, b}
$$

whenever $T^{\prime} \leq T$. 
(2) The $X_{\sigma, r a d}^{s, b}$ spaces interpolate nicely in the $s, b$ indices.

(3) For $b>\frac{1}{2}$, we have the following embedding

$$
\|u\|_{L_{t}^{\infty} H_{x}^{s}\left(\mathbb{R} \times B^{d}\right)} \leq C\|u\|_{X_{\sigma, r a d}^{s, b}\left(\mathbb{R} \times B^{d}\right)} .
$$

(4) An embedding that will be used frequently in this paper

$$
X_{\sigma, \mathrm{rad}}^{0, \frac{1}{4}} \hookrightarrow L_{t}^{4} L_{x}^{2}
$$

Note that

$$
\|f\|_{L_{t}^{4} L_{x}^{2}}=\left\|S_{\sigma}(t) f\right\|_{L_{t}^{4} L_{x}^{2}} \leq\left\|S_{\sigma}(t) f\right\|_{H_{t, \text { rad }}^{\frac{1}{4}} L_{x}^{2}}=\|f\|_{X_{\sigma, \text { rad }}^{0, \frac{1}{4}}} .
$$

Lemma 2.3 Let $b, s>0$ and $u_{0} \in H_{\text {rad }}^{s}\left(B^{d}\right)$. Then there exists $c>0$ such that for $0<T \leq 1$,

$$
\left\|S_{\sigma}(t) u_{0}\right\|_{X_{\sigma, r a d}^{s, b}\left((-T, T) \times B^{d}\right)} \leq c\left\|u_{0}\right\|_{H^{s}}
$$

The proofs of Lemma 2.2 and Lemma 2.3 can be found in [1].

We also recall the following lemma in $[4,26]$

Lemma 2.4 Let $0<b^{\prime}<\frac{1}{2}$ and $0<b<1-b^{\prime}$. Then for all $f \in X_{\sigma, \delta}^{s,-b^{\prime}}\left(B^{d}\right)$, we have the Duhamel term $w(t)=\int_{0}^{t} S_{\sigma}(t-s) f(\tau) d s \in X_{\sigma, \delta}^{s, b}\left(B^{d}\right)$ and moreover

$$
\|w\|_{X_{\sigma, \delta}^{s, b}\left(B^{d}\right)} \leq C \delta^{1+2 b-4 b^{\prime}}\|f\|_{X_{\sigma, \delta}^{s,-b^{\prime}}\left(B^{d}\right)} .
$$

From now on, for simplicity of notation, we write $H^{s}$ and $X_{\sigma}^{s, b}$ for the spaces $H_{\text {rad }}^{s}$ and $X_{\sigma, r a d}^{s, b}$ defined in this subsection.

\subsection{Useful inequalities}

Lemma 2.5 (Córdoba-Córdoba inequality $[17,18]$ ) Let $D \subset \mathbb{R}^{n}$ be a bounded domain with smooth boundary (resp. the $n$-dimensional torus). Let $\Delta$ be the Laplace operator on $D$ with Dirichlet boundary condition (rep. periodic condition). Let $\Phi$ be a convex $C^{2}(\mathbb{R}, \mathbb{R})$ satisfying $\Phi(0)=0$, and $\gamma \in[0,1]$. For any $f \in C^{\infty}(D, \mathbb{R})$, the inequality

$$
\Phi^{\prime}(f)(-\Delta)^{\gamma} f \geq(-\Delta)^{\gamma} \Phi(f)
$$

holds pointwise almost everywhere in $D$.

Lemma 2.6 (Complex Córdoba-Córdoba inequality) Let $D$ and $\Delta$ be as in Lemma 2.5. For any $f \in C^{\infty}(D, \mathbb{C})$, the inequality 


$$
2 \Re\left[f(-\Delta)^{\gamma} \bar{f}\right] \geq(-\Delta)^{\gamma}|f|^{2}
$$

holds pointwise almost everywhere in $D$.

Proof of Lemma 2.6 Let us write $f=a+\mathbf{i} b$ with real valued functions $a$ and $b$. We have

$$
\Re\left[(a+\mathbf{i} b)(-\Delta)^{\gamma}(a-\mathbf{i} b)\right]=a(-\Delta)^{\gamma} a+b(-\Delta)^{\gamma} b .
$$

Now we use Lemma 2.5 with $\Phi(x)=\frac{x^{2}}{2}$, we arrive at

$$
\Re\left[(a+\mathbf{i} b)(-\Delta)^{\gamma}(a-\mathbf{i} b)\right] \geq \frac{1}{2}(-\Delta)^{\gamma}\left(a^{2}+b^{2}\right)=\frac{1}{2}(-\Delta)^{\gamma}|f|^{2} .
$$

This finishes the proof of Lemma 2.6.

Corollary 2.7 Let $\gamma \in[0,1]$, we have, for $f \in C^{\infty}(D, \mathbb{C})$, that

$$
\left\langle(-\Delta)^{\gamma} f,|f|^{2} f\right\rangle \geq \frac{1}{2}\left\|(-\Delta)^{\frac{\gamma}{2}}|f|^{2}\right\|_{L^{2}}^{2}
$$

Lemma 2.8 (Radial Sobolev lemma on the unit ball) Let $\frac{1}{2}<s<\frac{d}{2}$. Then for any $f \in H^{s}:=H_{0}^{s}\left(B^{d}\right)^{3}$, we have

$$
|f(r)| \lesssim r^{s-\frac{d}{2}}\|f\|_{H^{s}} \quad \text { for all } r \in(0,1] .
$$

Proof of Lemma 2.8 Let $\frac{1}{2}<s<\frac{d}{2}$. We have for any $f \in H^{s}\left(\mathbb{R}^{d}\right.$ ) (see [14]), that

$$
|f(r)| \lesssim r^{s-\frac{d}{2}}\|f\|_{H^{s}\left(\mathbb{R}^{d}\right)} \quad \text { for all } r \in(0, \infty)
$$

On the other hand, since $B^{d}$ is a regular domain, we have the extension theorem (see for instance [34] and reference therein): there is a bounded operator $\mathfrak{E}: H_{0}^{s}\left(B^{d}\right) \rightarrow$ $H^{s}\left(\mathbb{R}^{d}\right)$ satisfying the following

(1) $\mathfrak{E} f(r)=f(r) \forall r \in[0,1]$, for any $f \in H_{0}^{s}\left(B^{d}\right)$;

(2) (continuity)

$$
\|\mathfrak{E} f\|_{H^{s}\left(\mathbb{R}^{d}\right)} \leq C\|f\|_{H_{0}^{s}\left(B^{d}\right)} .
$$

Therefore using (2.5), we notice that

$$
|\mathfrak{E} f(r)| \lesssim r^{s-\frac{d}{2}}\|\mathfrak{E} f\|_{H^{s}\left(\mathbb{R}^{d}\right)} \lesssim r^{s-\frac{d}{2}}\|f\|_{H_{0}^{s}\left(B^{d}\right)} \quad \text { for all } r \in(0, \infty) .
$$

\footnotetext{
${ }^{3} H_{0}^{s}\left(B^{d}\right)$ is the Sobolev space of order $s$ of functions $f: B^{d} \rightarrow \mathbb{C}$ vanishing on the boundary of $B^{d}$. 
Then for $r \in(0,1]$, we use the item (2.5) above to arrive at

$$
|f(r)| \lesssim r^{s-\frac{d}{2}}\|f\|_{H^{s}}
$$

Hence we finish the proof of Lemma 2.8.

\section{Description of the Inviscid-Infinite dimensional (IID) limit}

The IID limit combines a fluctuation-dissipation argument and an abstract version of the Bourgain's invariant measure globalization [5].

We consider a Hamiltonian equation

$$
\partial_{t} u=J H^{\prime}(u)
$$

where $J$ is a skew-adjoint operator on suitable spaces, $H(u)$ is the hamiltonian function, $H^{\prime}$ is the derivative with respect to $u$ (or a function of $u$, for instance to its complex conjugate). We can, for simplicity, assume the following form for the Hamiltonian

$$
H(u)=E_{K}(u)+E_{p}(u),
$$

where $E_{K}$ and $E_{p}$ refer to the kinetic energy of the system (having a quadratic power) and the potential energy, respectively. In the sequel we construct a general framework allowing to apply the Bourgain's invariant measure argument to the context of a general probability measure (not necessarily Gaussian based). We then present the strategy of the construction of the required measures.

\subsection{Abstract version of Bourgain's invariant measure argument}

Let $\Pi^{N}$ be the projection on the $N$-dimensional space $E^{N}$ spanned by the first $N$ eigenfunctions $\left\{e_{1}, \cdots, e_{N}\right\}$. Consider the Galerkin projections of (3.1)

$$
\partial_{t} u=\Pi^{N} J H^{\prime}(u)
$$

The equation (3.1) will be seen as (3.2) with $N=\infty$.

Assumption 1 (Uniform local well-posedness) The equation (3.2) is uniformly (in $N$ ) locally wellposed in the Cauchy-Hadamard sense on some Sobolev space $H^{s}$. And there is a function $f$ independent of $N$, such that for any given data $u_{0} \in \Pi^{N} H^{s}$, the time existence $T\left(u_{0}\right)$ of the corresponding solution $u$ is at least $f\left(\left\|u_{0}\right\|_{H^{s}}\right)$. Also, the following estimate holds

$$
\|u(t)\|_{H^{s}} \leq 2\left\|u_{0}\right\|_{H^{s}} \quad \text { for all }|t| \leq T\left(u_{0}\right)
$$

We denote by $\phi_{t}^{N}$ the local flow of (3.2), for $N=\infty$ we set $\phi_{t}=\phi_{t}^{\infty}$. For $N<\infty$, we assume that $\phi_{t}^{N}$ is defined globally in time. 
Remark 3.1 For the power-like nonlinear Schrödinger equation, $f(x)$ is of the form $x^{r}$ where $r$ is related to the power of the nonlinearity.

Assumption 2 (Convergence) For any $u_{0} \in H^{s}$, any sequence $\left(u_{0}^{N}\right)_{N}$ such that

(1) for any $N, u_{0}^{N} \in \Pi^{N} H^{s}$;

(2) $\left(u_{0}^{N}\right)$ converges to $u_{0}$ in $H^{s}$;

then $\phi_{t}^{N} u^{N}$ converges $\phi_{t} u$ in $C_{t} H^{s-}$.

Assumption 3 (Invariant measure) For any $N$, there is a measure $\mu_{N}$ invariant under the projection equations (3.2) and satisfying

(1) There is an increasing one-to-one function $g: \mathbb{R} \rightarrow \mathbb{R}_{+}$and a function $h: \mathbb{R}_{+} \rightarrow$ $\mathbb{R}_{+}$such that we have the uniform bound

$$
\int_{E^{N}} g\left(\|u\|_{H^{s}}\right) h\left(\|u\|_{H^{s_{0}}}\right) \mu^{N}(d u) \leq C,
$$

where $C>0$ is independent of $N$ and $s_{0}$ is some fixed number. We then have

$$
\int_{E^{N}} e^{\ln \left(1+g\left(\|u\|_{H^{s}}\right)\right)} h\left(\|u\|_{H^{s_{0}}}\right) \mu^{N}(d u) \leq 1+C .
$$

We set $\tilde{g}\left(\|u\|_{H^{s}}\right)=\ln \left(1+g\left(\|u\|_{H^{s}}\right)\right){ }^{4}$

(2) The following limit holds

$$
\lim _{i \rightarrow \infty} e^{-2 i} \sum_{j \geq 0} \frac{e^{-j}}{f \circ \tilde{g}^{-1}(2(i+j))}=0 .
$$

We set the number

$$
\kappa(i):=e^{-2 i} \sum_{j \geq 0} \frac{e^{-j}}{f \circ \tilde{g}^{-1}(2(i+j))} .
$$

Remark 3.2 For the case of Gibbs measures for NLS like equations, $g(x)$ is of the form $e^{a x^{2}}, a>0$ and $h(x)$ is a constant.

The Prokhorov theorem (Theorem A.4) combined with the estimate (3.3) implies the existence of a probability measure $\mu \in \mathfrak{p}\left(H^{s}\right)$ as an accumulation point when $N \rightarrow \infty$ for the measures $\left\{\mu^{N}\right\}$.

Theorem 3.3 Under the Assumptions 1, 2, and 3, there is a set $\Sigma \subset H^{s}$ such that

(1) $\mu(\Sigma)=1$

(2) The equation (3.1) is GWP on $\Sigma$

(3) The measure $\mu$ is invariant under the flow $\phi_{t}$ induced by the GWP;

\footnotetext{
${ }^{4}$ Remark that $\tilde{g}$ is increasing and one-to-one from $\mathbb{R}$ to $\mathbb{R}$.
} 
(4) For every $u_{0} \in \Sigma$, we have that

$$
\left\|\phi_{t} u_{0}\right\|_{H^{s}} \leq C\left(u_{0}\right) \tilde{g}^{-1}(1+\ln (1+|t|)) .
$$

The following is devoted to the proof of Theorem 3.3.

\subsubsection{Individual bounds}

Fix an arbitrary (small) number $a>0$, define the set

$$
E_{a}^{N}=\left\{u \in E^{N} \mid h\left(\|u\|_{H^{s_{0}}}\right) \geq a\right\}
$$

Proposition 3.4 Let $r \leq s, N \geq 1, i \geq 1$, there exists a set $\Sigma_{r, i}^{N} \subset E_{a}^{N}$ such that

$$
\mu^{N}\left(E_{a}^{N} \backslash \Sigma_{r, i}^{N}\right) \leq C a^{-1} \kappa(i),
$$

and for any $u_{0} \in \Sigma_{r, i}^{N}$, we have

$$
\left\|\phi_{t}^{N} u_{0}\right\|_{H^{r}} \leq 2 \tilde{g}^{-1}(1+i+\ln (1+|t|)) \text { for all } t \in \mathbb{R} \text {. }
$$

Moreover, we have the properties

$$
\Sigma_{s, i}^{N_{1}} \subset \Sigma_{s, i}^{N_{2}} \text { if } N_{1} \leq N_{2} ; \quad \Sigma_{s, i_{1}}^{N} \subset \Sigma_{s, i_{2}}^{N} \text { if } i_{1} \leq i_{2} ; \quad \Sigma_{s_{1}, i}^{N} \subset \Sigma_{s_{2}, i}^{N} \text { if } s_{1} \geq s_{2} .
$$

Proof of Proposition 3.4 It suffices to consider $t>0$. We define the sets

$$
B_{r, i, j}^{N}:=\left\{u \in E_{a}^{N} \mid\|u\|_{H^{r}} \leq \tilde{g}^{-1}(2(i+j))\right\} .
$$

Let $T_{0}$ be the local time existence on $B_{r, i, j}^{N}$. From the local well-posedness, we have for $t \leq T_{0}=f\left(\tilde{g}^{-1}(2(i+j))\right)$ (see Assumption 1), that

$$
\phi_{t}^{N} B_{r, i, j}^{N} \subset\left\{u \in E_{a}^{N} \mid\|u\|_{H^{r}} \leq 2 \tilde{g}^{-1}(2(i+j))\right\} .
$$

Now, let us define the set

$$
\Sigma_{r, i, j}^{N}:=\bigcap_{k=0}^{\left[\frac{e^{j}}{T_{0}}\right]} \phi_{-k T_{0}}^{N}\left(B_{r, i, j}^{N}\right) .
$$

We use (3.3) and the Chebyshev inequality to obtain the following

$$
\mu^{N}\left(E_{a}^{N} \backslash \Sigma_{r, i, j}^{N}\right)=\mu^{N}\left(\left\{u \in E_{a}^{N} \mid\|u\|_{H^{r}} \geq \tilde{g}^{-1}(2(i+j))\right\}\right)
$$




$$
\begin{aligned}
& \lesssim e^{-2(i+j)}\left[\frac{e^{j}}{T_{0}}\right] \int_{E_{a}^{N}} e^{\tilde{g}\left(\|u\|_{\left.H^{r}\right)} \mu^{N}(d u)\right.} \\
& \lesssim e^{-2(i+j)}\left[\frac{e^{j}}{T_{0}}\right] a^{-1} \int_{L^{2}} e^{\tilde{g}\left(\|u\|_{H^{r}}\right)} h\left(\|u\|_{H^{s_{0}}}\right) \mu^{N}(d u) \\
& \lesssim a^{-1}\left[\frac{e^{j}}{T_{0}}\right] e^{-2(i+j)} \\
& \lesssim a^{-1} \frac{e^{-2 i} e^{-j}}{f \circ \tilde{g}^{-1}(2(i+j))}
\end{aligned}
$$

Let us finally define

$$
\Sigma_{r, i}^{N}=\bigcap_{j \geq 0} \Sigma_{r, i, j}^{N}
$$

We then obtain

$$
\mu^{N}\left(E_{a}^{N} \backslash \Sigma_{s, i}^{N}\right) \leq C a^{-1} e^{-2 i} \sum_{j \geq 0} \frac{e^{-j}}{f \circ \tilde{g}^{-1}(i+j)}=C a^{-1} \kappa(i) .
$$

We claim that for $u_{0} \in \Sigma_{r, i, j}^{N}$, the following estimate holds

$$
\left\|\phi_{t}^{N} u_{0}\right\|_{H^{r}} \leq 2 \tilde{g}^{-1}(2(i+j)) \text { for all } t \leq h(j) .
$$

Indeed we write $t=k T_{0}+\tau$ where $k$ is an integer in $\left[0, \frac{e^{j}}{T_{0}}\right]$ and $\tau \in\left[0, T_{0}\right]$. We have

$$
\phi_{t}^{N} u_{0}=\phi_{\tau+k T_{0}}^{N} u_{0}=\phi_{\tau}^{N}\left(\phi_{k T_{0}}^{N} u_{0}\right) \text {. }
$$

Now, recall that $u_{0} \in \phi_{-k T_{0}}^{N}\left(B_{r, i, j}^{N}\right)$, then $u_{0} \in \phi_{-k T_{0}}^{N} w$ where $w \in B_{r, i, j}^{N}$. Then $\phi_{t}^{N} u_{0}=\phi_{\tau}^{N} w$.

For $t>0$, there $j \geq 0$ such that

$$
e^{j-1} \leq 1+t \leq e^{j}
$$

Then

$$
j \leq 1+\ln (1+t) .
$$

We arrive at

$$
\left\|\phi_{t}^{N} u_{0}\right\|_{H^{r}} \leq 2 \tilde{g}^{-1}(1+i+\ln (1+t)) .
$$

The properties (3.7) follow easily from the definition of the sets $\Sigma_{r, i}^{N}$. Remark that the definition of $\Sigma_{r, i}^{N}$ does not depend on the measure $\mu^{N}$ but just on the functions 
$h$ and $g$, as can be noticed in (3.4), (3.8), (3.9) and (3.10). If $N_{1} \leq N_{2}$, then we see trivially that $B_{r, i, j}^{N_{1}} \subset B_{r, i, j}^{N_{2}}$, it then follows from (3.9) and (3.10) that $\Sigma_{r, i}^{N_{1}} \subset \Sigma_{r, i}^{N_{2}}$. Using the fact that $g^{-1}$ is increasing, we obtain $\Sigma_{s, i_{1}}^{N} \subset \Sigma_{s, i_{2}}^{N}$ for $i_{1} \leq i_{2}$. Similarly, the inequality $\|u\|_{H^{s_{2}}} \leq\|u\|_{H^{s_{1}}}$ for $s_{1} \geq s_{2}$ leads to remaining inclusion. The proof of Proposition 3.4 is finished.

\subsubsection{Statistical ensemble}

We want to pass to the limit $N \rightarrow \infty$ in the sets $\Sigma_{r, i}^{N}$. We introduce the following limiting set:

$$
\Sigma_{r, i}:=\left\{u \in H^{r} \mid \exists\left(u^{N_{k}}\right)_{k}, \lim _{k \rightarrow \infty} u^{N_{k}}=u, \text { where } u^{N_{k}} \in \Sigma_{s, i}^{N_{k}}\right\}
$$

We then define the following set

$$
\Sigma_{r}=\bigcup_{i \geq 1} \overline{\Sigma_{r, i}}
$$

Let $\left(l_{k}\right)$ be a sequence such that $\lim _{k \rightarrow \infty} l_{k}=s$, the statistical ensemble is given by

$$
\Sigma=\bigcap_{k \in l_{k}} \Sigma_{k}
$$

Proposition 3.5 The set $\Sigma$ constructed above is of full $\mu$-measure:

$$
\mu(\Sigma)=1
$$

Proof of Proposition 3.5 Let us first observe that

$$
\Sigma_{r, i}^{N} \subset \Sigma_{r, i}
$$

because for any $u \in \Sigma_{r, i}^{N}$ the constant sequence $u_{N}=u$ converges to $u$, and then $u \in \Sigma_{r, i}$.

Now by the Portmanteau theorem (see Theorem A.3) and the use of the bound (3.5) we have that

$$
\begin{aligned}
& \mu\left(\overline{\Sigma_{r, i}}\right) \geq \lim _{N_{k} \rightarrow \infty} \mu^{N_{k}}\left(\overline{\Sigma_{r, i}}\right) \\
& \left.\quad \geq \lim _{N_{k} \rightarrow \infty} \mu^{N_{k}} \overline{\left(\Sigma_{r, i}^{N_{k}}\right.}\right) \\
& \geq \lim _{N_{k} \rightarrow \infty} \mu^{N_{k}}\left(\Sigma_{s, i}^{N_{k}}\right) \geq \lim _{N_{k} \rightarrow \infty}\left(1-C a^{-1} \kappa(i)\right)=1-C a^{-1} \kappa(i) .
\end{aligned}
$$


On the other hand, from the properties (3.7), we have that $\left(\overline{\Sigma_{r, i}}\right)_{i \geq 1}$ is non-decreasing. In particular,

$$
\mu\left(\cup_{i \geq 1} \overline{\Sigma_{r, i}}\right)=\lim _{i \rightarrow \infty} \mu\left(\overline{\Sigma_{r, i}}\right)
$$

Therefore

$$
\mu\left(\cup_{i \geq 1} \overline{\Sigma_{r, i}}\right)=\lim _{i \rightarrow \infty} \mu\left(\overline{\Sigma_{r, i}}\right) \geq \lim _{i \rightarrow \infty}\left(1-C a^{-1} \kappa(i)\right)=1
$$

Since $\mu$ is a probability measure, we obtain

$$
1 \geq \mu\left(\Sigma_{r}\right) \geq 1
$$

This finishes the proof of Proposition 3.5.

\subsubsection{Globalization}

Proposition 3.6 Let $r \leq s$. For any $u_{0} \in \Sigma_{r}$, there is a unique global solution $u \in$ $C_{t} H^{s}$ such that

$$
\left\|\phi_{t} u_{0}\right\|_{H^{s}} \leq 2 \tilde{g}^{-1}(1+i+\ln (1+|t|)) \quad \forall t \in \mathbb{R}
$$

Proof of Proposition 3.6 For $u_{0} \in \Sigma_{r} \cap \operatorname{supp}(\mu)$ (recall that $\mu\left(\Sigma_{r}\right)=1$ ), we have that

(1) $u_{0} \in H^{s}$ since $\operatorname{supp}(\mu) \subset H^{s}$;

(2) there is $i \geq 1$ such that $u_{0} \in \overline{\Sigma_{r, i}}$.

We will consider that $u_{0} \in \Sigma_{r, i}$, the case $u_{0} \in \partial \Sigma_{r, i}$ can be obtained by a limiting argument (see [54]). By construction of $\Sigma_{r, i}$, there is $N_{k} \rightarrow \infty$ such that

$$
u_{0}=\lim _{N_{k} \rightarrow \infty} u^{N_{k}}, \quad u^{N_{k}} \in \Sigma_{r, i}^{N_{k}}
$$

Now using (3.6), we have

$$
\left\|\phi_{t}^{N_{k}} u^{N_{k}}\right\|_{H^{r}} \leq 2 \tilde{g}^{-1}(1+i+\ln (1+|t|))
$$

this gives in particular that, at $t=0$,

$$
\left\|u^{N_{k}}\right\|_{H^{r}} \leq 2 \tilde{g}^{-1}(1+i)
$$

A passage to the limit $N_{k} \rightarrow \infty$ shows that

$$
\left\|u_{0}\right\|_{H^{r}} \leq 2 \tilde{g}^{-1}(1+i) .
$$


Now for any fixed $T>0$, set $b=2 \tilde{g}^{-1}(1+i+\ln (1+|T|))$ and $R=b+1$, so that $u^{N_{k}}, u_{0} \in B_{R}\left(H^{r}\right)$. Let $T_{R}$ be the local existence time of $B_{R}$. We have that $\phi_{t}$ and $\phi_{t}^{N_{k}} u^{N_{k}}$ exist for $|t| \leq T_{R}$. Using Assumption 2, we have for $r<s$,

$$
\left\|\phi_{t} u_{0}\right\|_{L_{t}^{\infty} H^{r}} \leq b \leq R
$$

This bound tells us that after the time $T_{R}$ the solution stays in the same ball as the initial data. We can then iterate on intervals $\left[n T_{R},(n+1) T_{R}\right]$ still consuming the fixed time $T$. Since this time is arbitrary, we conclude to the global existence. The estimate (3.11) follows easily a passage to the limit from (3.6). Now the proof of Proposition 3.6 is finished.

Remark 3.7 It follows from the proof above that, if $u_{0} \in \Sigma_{r}$ we have that

$$
\lim _{k \rightarrow \infty}\left\|\phi_{t} u_{0}-\phi_{t}^{N_{k}} u^{N_{k}}\right\|_{H^{r}}=0
$$

where $u^{N_{k}} \in \Sigma_{r, i}^{N_{k}}$ and $\lim _{k \rightarrow \infty}\left\|u_{0}-u^{N_{k}}\right\|_{H^{r}}=0$.

\subsubsection{Invariance results}

Theorem 3.8 The measure $\mu$ is invariant under $\phi_{t}$.

Proof of Theorem 3.8 It follows from the Skorokhod representation theorem (Theorem A.5) the existence of $u_{0}^{N}$ a random variable distributed by $\mu^{N}$ and $u_{0}$ a one distributed by $\mu$ such that

$$
u_{0}^{N} \rightarrow u_{0} \quad \text { as } N \rightarrow \infty, \quad \text { almost surely. }
$$

Combining this with the Assumption 2, we then have the almost sure convergence

$$
\phi_{t}^{N} u_{0}^{N}(\omega) \rightarrow \phi_{t} u_{0}(\omega) \quad \text { on } H^{s-}
$$

For any fixed $t$, we know that the distribution $\mathscr{D}\left(\phi_{t}^{N} u_{0}^{N}\right)$ is $\mu^{N}$, by the invariance of $\mu^{N}$ under (3.2). Let us look at $\phi_{t}^{N} u_{0}^{N}$ and $\phi_{t} u_{0}$ as $H^{s-}-$ valued random variables. We then have the following two results:

(1) The pointwise convergence in (3.13) implies that $\mu^{N}$ weakly converges to $\mathscr{D}\left(\phi_{t} u_{0}\right)$ for any fixed $t$, hence the invariance of $\mathscr{D}\left(\phi_{t} u_{0}\right)$ because $\mu^{N}$ does not depend on $t$

(2) We already know that $\mu^{N}$ converges weakly to $\mu$.

The proof of Theorem 3.8 is complete.

Assumption 4 (Second conservation law) The equation (3.1) admits a conservation law of the form 


$$
M(u)=\frac{1}{2}\|u\|_{H^{\gamma}}^{2},
$$

where $\gamma<s$.

Theorem 3.9 Under the Assumption 4 we have the following

$$
\phi_{t} \Sigma=\Sigma \text {. }
$$

The proof of Theorem 3.9 relies on the lemma 3.10 below. This lemma roughly states that the evolution under $\phi_{t}^{N}$ of a set $\Sigma_{r, i}^{N}$ is a set of the same kind, giving then the essential mechanism needed for the invariance. The remaining part relies on the definition of the set $\Sigma$ and on a limiting argument.

Lemma 3.10 Let $r \leq s$, for any $\gamma<r_{1}<r$ and any $t$, there exists $i_{1}:=i_{1}(t)$ such that for any $i$, if $u_{0} \in \Sigma_{s, i}^{N}$ then $\phi_{t}^{N} u_{0} \in \Sigma_{r_{1}, i+i_{1}}^{N}$.

Proof of Lemma 3.10 We can assume without loss of generality that $t \geq 0$. For $u_{0} \in$ $\Sigma_{r, i}^{N}$, we have, by definition, that

$$
\left\|\phi_{t_{1}}^{N} u_{0}\right\|_{H^{r}} \leq 2 \tilde{g}^{-1}(i+j) \quad \forall t_{1} \leq e^{j}
$$

Let us pick a function $i_{1}:=i_{1}(t)$ satisfying $e^{j}+t \leq e^{i_{1}+j}$ for any $j \geq 0$. Then

$$
\left\|\phi_{t_{1}+t}^{N} u_{0}\right\|_{H^{r}} \leq 2 \tilde{g}^{-1}\left(i+i_{1}+j\right) \quad \forall t+t_{1} \leq e^{j+i_{1}} .
$$

But for $t+t_{1} \leq e^{j+i_{1}}$, one readily has $t_{1} \leq e^{j+i_{1}}-t$. On the other hand we have, from the definition of $i_{1}$, that $e^{j+i_{1}}-t \geq e^{\bar{j}}$. From these two facts, we obtain

$$
\left\|\phi_{t_{1}+t}^{N} u_{0}\right\|_{H^{r}} \leq 2 \tilde{g}^{-1}\left(i+i_{1}+j\right) \quad \forall t_{1} \leq e^{j} .
$$

Since $u_{0} \in \Sigma_{i, s}^{N}$, using the estimate (3.6), we have

$$
\left\|\phi_{t}^{N} u_{0}\right\|_{H^{r}} \leq 2 \tilde{g}^{-1}(1+i+\ln (1+|t|))
$$

Hence, using Assumption 4, we have

$$
\left\|u_{0}\right\|_{H^{\gamma}} \leq\left\|u_{0}\right\|_{H^{r}} \leq 2 \tilde{g}^{-1}(1+i)
$$

Using the conservation of the $H^{\gamma}$-norm (Assumption 4), we obtain

$$
\left\|\phi_{t+t_{1}}^{N} u_{0}\right\|_{H^{\gamma}} \leq 2 \tilde{g}^{-1}(1+i) .
$$

For $\gamma<r_{1}<r$, by interpolation there is $0<\theta<1$ such that

$$
\left\|\phi_{t+t_{1}}^{N} u_{0}\right\|_{H^{r_{1}}} \leq\left\|\phi_{t+t_{1}}^{N} u_{0}\right\|_{H^{\gamma}}^{1-\theta}\left\|\phi_{t+t_{1}}^{N} u_{0}\right\|_{H^{s_{1}}}^{\theta} \leq 2(\tilde{g}(1+i))^{1-\theta}\left(\tilde{g}^{-1}\left(i+i_{1}+j\right)\right)^{\theta} .
$$


As $\tilde{g}^{-1}$ is increasing, we take $i_{1}$ large enough to obtain the bound

$$
\left\|\phi_{t+t_{1}}^{N} u_{0}\right\|_{H^{r_{1}}} \leq \tilde{g}^{-1}\left(i+i_{1}+j\right) \quad \forall t_{1} \leq e^{j} .
$$

Thus $\phi_{t+t_{1}}^{N} u_{0} \in B_{r_{1}, i+i_{1}, j}^{N}$ for all $t_{1} \leq e^{j}$, for all $j \geq 0$. We then have $\phi_{t}^{N} u_{0} \in \Sigma_{r_{1}, i+i_{1}}^{N}$. Then the proof of Lemma 3.10 is finished.

Remark 3.11 In some situation, Assumption 4 is not necessary. If the control on the $H^{S}$-norm provides a control on the energy, we can proof Lemma 3.10 without any supplementary conservation law.

Now let us finish the proof of Theorem 3.9. Since any $\Sigma_{r}$ is of full $\mu-$ measure and the intersection is countable, we obtain the first statement.

Let us take $u_{0} \in \Sigma$, then $u_{0}$ belong to each $\Sigma_{r}, r \in l$. First, consider $u_{0} \in \Sigma_{i_{r}}$. There is $i \geq 1$ such that $u_{0} \in \Sigma_{i_{r}}$, therefore $u_{0}$ is the limit of a sequence $\left(u_{0}^{N}\right)$ such that $u_{0}^{N} \in \Sigma_{i, r}^{N}$ for every $N$. Now thanks to Lemma 3.10, there is $i_{1}:=i_{1}(t)$ such that $\phi_{t}^{N}\left(u_{0}^{N}\right) \in \Sigma_{i+i_{1}, r_{1}}^{N}$. Using the convergence (3.12), we see that $\phi^{t}\left(u_{0}\right) \in \Sigma_{i+i_{1}, r_{1}}$. Now if $u_{0} \in \partial \Sigma_{i, r}$, there is $\left(u_{0}^{k}\right)_{k} \subset \Sigma_{i, r}$ that converges to $u_{0}$ in $H^{r}$. Since we showed that $\phi_{t} \Sigma_{i, r} \subset \Sigma_{i+i_{1}, r_{1}}$ and $\phi_{t}(\cdot)$ is continuous, we see that $\phi_{t}\left(u_{0}\right)=\lim _{k} \phi_{t}\left(u_{0}^{k}\right) \in$ $\overline{\Sigma_{i+i_{1}, r_{1}}}$. One arrives at $\phi_{t} \overline{\Sigma_{i, r}} \subset \overline{\Sigma_{i+i_{1}, r_{1}}} \subset \Sigma_{r_{1}}$. Hence $\phi_{t} \Sigma \subset \Sigma$.

Now, let $u$ be in $\Sigma$. Since $\phi_{t}$ is well-defined on $\Sigma$ we can set $u_{0}=\phi_{-t} u$, we then have $u=\phi_{t} u_{0}$, hence $\Sigma \subset \phi_{t} \Sigma$.

\subsection{Construction of the required invariant measures}

Here we present a strategy to achieve the fulfillment of Assumption 3. It is based on a fluctuation-dissipation method.

Let us introduce a probabilistic setting and some notations that are going to be used from this section on. First, $(\Omega, \mathscr{F}, \mathbb{P})$ is a complete probability space. If $E$ is a Banach space, we can define random variables $X: \Omega \rightarrow E$ as Bochner measurable functions with respect to $\mathscr{F}$ and $\mathscr{B}(E)$, where $\mathscr{B}(E)$ is the Borel $\sigma-$ algebra of $E$.

For every positive integer $N$, we denote by $W^{N}$ an $N$-dimensional Brownian motion with respect to the filtration $\left(\mathscr{F}_{t}\right)_{t \geq 0}$.

Throughout the sequel, we use $\langle\cdot, \cdot\rangle$ as the real dot product in $L^{2}$ :

$$
\langle u, v\rangle=\operatorname{Re} \int u \bar{v} d x, \quad \text { for all } u, v \in L^{2} .
$$

Ocassionally this notation is used to write a duality bracket when the context is not confusing.

We set the fluctuation-dissipation equation

$$
\partial_{t} u=\Pi^{N} J H^{\prime}(u)-\alpha \mathscr{L}(u)+\sqrt{\alpha} d W^{N},
$$

where $\mathscr{L}(u)$ is a dissipation operator. 
Assumption 5 (Exponential moment) We assume that $\mathscr{L}(u)$ satisfies the dissipation inequality

$$
\mathscr{E}(u)=E^{\prime}(u, \mathscr{L}(u)) \geq e^{\xi^{-1}\left(\|u\|_{H^{s}}\right)}\|u\|_{H^{s_{0}}}^{2},
$$

where $\xi^{-1}(x) \geq x$ is a one-to-one convex function from $\mathbb{R}_{+}$to $\mathbb{R}_{+}$.

We assume also that the (local existence) function $f$ defined in Assumption 1 is of the form $C x^{-r}$ for some $r>0$.

Assumption 6 The equation (3.14) is stochastically globally well-posed on $E^{N}$, that is:

For every $\mathscr{F}_{0}$-measurable random variable $u_{0}$ in $E_{N}$, we have

(1) for $\mathbb{P}$-almost all $\omega \in \Omega$, (3.14) with initial condition $u(0)=\Pi^{N} u_{0}^{\omega}$ has a unique global solution $u_{\alpha}^{N}\left(t ; u_{0}^{\omega}\right)$;

(2) if $u_{0, n}^{\omega} \rightarrow u_{0}^{\omega}$ then $u_{\alpha}^{N}\left(\cdot ; u_{0, n}^{\omega}\right) \rightarrow u_{\alpha}^{N}\left(\cdot ; u_{0}^{\omega}\right)$ in $C_{t} E^{N}$;

(3) the solution $u_{\alpha}^{N}$ is adapted to $\left(\mathscr{F}_{t}\right)$.

Under Assumption (6), we can introduce the transition probability

$$
T_{t, \alpha}^{N}(v, \Gamma)=\mathbb{P}\left(u_{\alpha}^{N}(t ; v) \in \Gamma\right) \quad \text { where } v \in L^{2} \text { and } \Gamma \in \operatorname{Bor}\left(L^{2}\right) .
$$

We then define the Markov operators

$$
\begin{aligned}
& P_{t, \alpha}^{N} f(v)=\int_{L^{2}} f(w) T_{t, \alpha}^{N}(v, d w) \quad L^{\infty}\left(L^{2}, \mathbb{R}\right) \rightarrow L^{\infty}\left(L^{2}, \mathbb{R}\right) \\
& P_{t, \alpha}^{N *} \lambda(\Gamma)=\int_{L^{2}} \lambda(d w) T_{t, \alpha}^{N}(w, \Gamma) \quad \mathfrak{p}\left(L^{2}\right) \rightarrow \mathfrak{p}\left(L^{2}\right) .
\end{aligned}
$$

Let us remark that

$$
P_{t, \alpha}^{N} f(v)=\mathbb{E} f\left(u_{\alpha}^{N}(t, v)\right)
$$

Using the continuity of the solution $u_{\alpha}^{N}(t, \cdot)$ in the second variable (the initial data), we observe the Feller property:

$$
P_{t, \alpha}^{N} C_{b}\left(L^{2}, \mathbb{R}\right) \subset C_{b}\left(L^{2}, \mathbb{R}\right) .
$$

Here $C_{b}\left(L^{2}, \mathbb{R}\right)$ is the space of continuous bounded functions $f: L^{2} \rightarrow \mathbb{R}$.

Let us also define the Markov groups associated to (3.2), recall that in Assumption (1) we assume that $\phi_{t}^{N}$ exists globally in $t$. The associated Markov groups are the following

$$
\begin{aligned}
P_{t}^{N} f(v) & =f\left(\phi_{t}^{N} \Pi^{N} v\right) \quad C_{b}\left(L^{2}, \mathbb{R}\right) \rightarrow C_{b}\left(L^{2}, \mathbb{R}\right) \\
P_{t}^{N *} \lambda(\Gamma) & =\lambda\left(\phi_{-t}^{N}(\Gamma)\right) \quad \mathfrak{p}\left(L^{2}\right) \rightarrow \mathfrak{p}\left(L^{2}\right) .
\end{aligned}
$$


Combining the Itô formula, the Krylov-Bogoliubov argument (Lemma A.9), the Prokhorov theorem (Theorem A.4) and Lemma A.10 we obtain the following:

Theorem 3.12 Under Assumptions 5 and 6 , let $N \geq 1$, there is an stationary measure $\mu_{\alpha}^{N}$ for (3.14) such that

$$
\int_{L^{2}} e^{\xi^{-1}\left(\|u\|_{H^{s}}\right)}\|u\|_{H^{s_{0}}}^{2} \mu_{\alpha}^{N}(d u) \leq \int_{L^{2}} \mathscr{E}(u) \mu_{\alpha}^{N}(d u) \leq C,
$$

where $C$ does not depend on $(N, \alpha)$.

There is a subsequence $\left\{\alpha_{k}\right\}$ such that

$$
\lim _{k \rightarrow \infty} \mu_{\alpha_{k}}^{N}=\mu^{N} \text { weakly, for any } N
$$

Assumption 7 (Inviscid limit) The following convergence holds

$$
\lim _{k \rightarrow \infty} P_{t, \alpha_{k}}^{N *} \mu_{\alpha_{k}}^{N}=P_{t}^{N *} \mu^{N} \quad \text { weakly, for any } N
$$

Theorem 3.13 Under Assumptions 5 and 7, the measures $\mu^{N}$ are invariant under (3.2) respectively. They satisfy

$$
\int_{L^{2}} e^{\xi^{-1}\left(\|u\|_{H^{s}}\right)}\|u\|_{H^{s_{0}}}^{2} \mu^{N}(d u) \leq \int_{L^{2}} \mathscr{E}(u) \mu^{N}(d u) \leq C,
$$

where $C$ is independent of $N$.

Moreover, we have that

$$
\lim _{i \rightarrow \infty} e^{-2 i} \sum_{j \geq 0} \frac{e^{-j}}{f \circ \xi(2(i+j))}=0
$$

Proof of Theorem 3.13 For simplicity we write the measure $\mu_{\alpha_{k}}^{N}$ constructed above as $\mu_{k}^{N}$. The invariance of $\mu^{N}$ under (3.2) follows from the following diagram

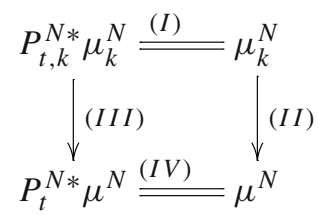

The step (I) is the stationarity of $\mu_{k}^{N}$ under (3.14), the step (II) describes the weak convergence of $\left(\mu_{k}^{N}\right)$ to $\mu^{N}$. Therefore, with the help of the weak convergence in (III) (Assumption 7), we obtain the desired invariance in $(I V)$. 
As for the proof of (3.17), we first remark that the weak convergence $\mu_{k}^{N} \rightarrow \mu^{N}$ combined with the lower semi-continuity of $\mathscr{E}(u)$ yields

$$
\int_{L^{2}} e^{\xi^{-1}\left(\|u\|_{H^{s}}\right)}\|u\|_{H^{s_{0}}}^{2} \mu^{N}(d u) \leq \int_{L^{2}} \mathscr{E}(u) \mu^{N}(d u) \leq C,
$$

with $C$ is the same constant in (3.16), in particular $C$ does not depend on $N$.

Now using the form of $f$, we have that

$$
f \circ \xi(i+j)=C(\xi(i+j))^{-r} .
$$

Now recall that $\xi^{-1} \geq x$, then $\xi \leq x^{-1}$. So, we obtain $f \circ \xi(2(i+j)) \gtrsim(i+j)^{-r}$, which leads readily to (3.18). Then the proof of Theorem 3.13 is finished.

From now, Assumption 3 is disintegrated into Assumptions 5 to 7.

\section{Proof of Theorem 1.1: Probabilistic part}

In this section, we present the probabilistic part of the proof of Theorem 1.1, that is, the fulfillment of Assumptions 4 to 7. First, we remark that Assumption 3 was decomposed in the strategy given in Sect. 3.2 into Assumptions 5 to 7. Assumptions 1 and 2 are of deterministic type and will be given in Sect. 5. We also prepare, simultaneously, the setting for the proof of Theorem 1.3. Assumption 4 is given by the existence of a second conservation law (the $L^{2}$-norm).

Let $N$ be a positive integer, consider the Galerkin approximation

$$
\partial_{t} u=-\mathbf{i}\left((-\Delta)^{\sigma} u+\Pi^{N}|u|^{2} u\right) .
$$

We define a Brownian motion following the case we set the equation on the unit ball $B^{d}$ :

$$
W^{N}(t, x)=\sum_{n=1}^{N} a_{n} e_{n}(|x|) \beta_{n}(t)
$$

where $\left(e_{n}\right)_{n}$ is the sequence of eigenfunctions of radial Dirichlet Laplacian $-\Delta$ on $B^{d}$. Here $\left(\beta_{n}(t)\right)$ is a fixed sequence of independent one dimensional Brownian motions with filtration $\left(\mathscr{F}_{t}\right)_{t \geq 0}$. The numbers $\left(a_{m}\right)_{m \geq 1}$ are complex numbers such that $\left|a_{m}\right|$ decreases sufficiently fast to 0 . More precisely we assume that

$$
A_{r}:=\sum_{n \geq 1} z_{n}^{2 r}\left|a_{n}\right|^{2}<+\infty \text { for } r \leq \sigma
$$


where $\left(z_{n}^{2}\right)$ are the eigenvalues of $-\Delta$ associated to $\left(e_{n}\right)$. Set

$$
A_{r}^{N}=\sum_{n=1}^{N} z_{n}^{2 r}\left|a_{n}\right|^{2}
$$

Let us introduce the following fluctuation-dissipation model of (4.1). For $\sigma \in(0,1]$, we define a dissipation operator as follows

$$
\mathscr{L}(u)= \begin{cases}(-\Delta)^{s-\sigma} u+e^{\xi^{-1}\left(\|u\|_{H^{s-}}\right)} u, & \text { if } s>\frac{d}{2} \\ e^{\xi^{-1}\left(\|u\|_{H^{s-}}\right)}\left[(-\Delta)^{s-\sigma} u+\Pi^{N}|u|^{2} u\right] & \text { if } 0<s \leq 1+\sigma\end{cases}
$$

where $\xi: \mathbb{R}_{+} \rightarrow \mathbb{R}_{+}$is any one-to-one increasing concave function. We assume that $\xi^{-1}(x) \geq x$. We then write the stochastic equation

$$
d u=-\mathbf{i}\left((-\Delta)^{\sigma} u+\Pi^{N}|u|^{2} u\right) d t-\alpha \mathscr{L}(u) d t+\sqrt{\alpha} d W^{N}
$$

where $\alpha \in(0,1)$.

Remark 4.1 The setting of Theorem 1.3 is contained in the case $0<s \leq 1+\sigma$ :

$$
\mathscr{L}(u)=e^{\xi^{-1}\left(\|u\|_{H^{s-}}\right)}\left[(-\Delta)^{s-\sigma} u+\Pi^{N}|u|^{2} u\right] .
$$

Therefore the estimates obtained using that form of $\mathscr{L}(u)$ will also serve in the proof of Theorem 1.3 (see Sect. 6).

\subsection{Fulfillment of Assumption 5 (The dissipation inequality and time of local existence)}

For the polynomial nature of the time of local existence we refer to Theorem 5.1.

For the dissipation inequality, we set $\mathscr{E}(u):=E^{\prime}(u, \mathscr{L}(u))$. We have the following.

(1) For $s>\frac{d}{2}$,

$$
\mathscr{E}(u)=\|u\|_{H^{s}}^{2}+\left\langle(-\Delta)^{s-\sigma} u, \Pi^{N}|u|^{2} u\right\rangle+e^{\xi^{-1}\left(\|u\|_{H^{s-}}\right)}\left(\|u\|_{L^{4}}^{4}+\|u\|_{H^{\sigma}}^{2}\right) .
$$

Using Cauchy-Schwarz and Agmon's inequalities and the algebra property of $H^{s-}$ for $s>\frac{d}{2}$, we obtain

$$
\begin{aligned}
\left|\left\langle(-\Delta)^{s-\sigma} u, \Pi^{N}|u|^{2} u\right\rangle\right| & \leq\|u\|_{H^{s-\sigma}}\left\||u|^{2} u\right\|_{H^{s-\sigma}} \leq C\|u\|_{L^{\infty}}\left\||u|^{2} u\right\|_{H^{s-\sigma}} \\
& \leq C\|u\|_{L^{2}}^{1-\frac{d}{2 s}}\|u\|_{H^{s}}^{\frac{d}{2 s}}\left\||u|^{2} u\right\|_{H^{s-\sigma}} \leq C\|u\|_{H^{s}}\|u\|_{H^{s-}}^{3} \\
& \leq \tilde{C}+\frac{1}{2}\|u\|_{H^{s}}^{2}\|u\|_{H^{s-}}^{6} \leq \tilde{C}+\frac{1}{2} e^{\xi^{-1}\left(\|u\|_{H^{s-}}\right)\|u\|_{H^{s}}^{2}}
\end{aligned}
$$


Remark that the $\tilde{C}$ is an absolute constant. Overall,

$$
\mathscr{E}(u) \geq\|u\|_{H^{s}}^{2}+\frac{1}{2} e^{\xi^{-1}\left(\|u\|_{H^{s-}}\right)}\left(\|u\|_{L^{4}}^{4}+\|u\|_{H^{s}}^{2}\right)-\tilde{C} .
$$

(2) For $0<s \leq 1+\sigma$,

$$
\begin{aligned}
\mathscr{E}(u) & =e^{\xi^{-1}\left(\|u\|_{H^{s-}}\right)}\left(\|u\|_{H^{s}}^{2}+\left\langle(-\Delta)^{s-\sigma} u, \Pi^{N}|u|^{2} u\right\rangle\right. \\
& \left.+\left\langle\Pi^{N}|u|^{2} u,(-\Delta)^{\sigma} u\right\rangle+\left\|\Pi^{N}|u|^{2} u\right\|_{L^{2}}^{2}\right) .
\end{aligned}
$$

We are able to give a useful estimate of $\mathscr{E}(u)$ in the following two cases:

(a) For $\max \left(\frac{1}{2}, \sigma\right) \leq s \leq 1+\sigma$ :

In order the treat the term $\left\langle(-\Delta)^{s-\sigma} u, \Pi^{N}|u|^{2} u\right\rangle$ and $\left\langle\Pi^{N}|u|^{2} u,(-\Delta)^{\sigma} u\right\rangle$, we use the Córdoba-Córdoba inequality ( see Corollary 2.7) since $s-\sigma \in[0,1]$, $s$ being in $\left[\max \left(\frac{1}{2}, \sigma\right), 1+\sigma\right]$. We have the following

$$
\begin{aligned}
\left\langle(-\Delta)^{s-\sigma} u,|u|^{2} u\right\rangle & \geq \frac{1}{2}\left\|(-\Delta)^{\frac{s-\sigma}{2}}|u|^{2}\right\|_{L^{2}}^{2}, \\
\left\langle(-\Delta)^{\sigma} u,|u|^{2} u\right\rangle & \geq \frac{1}{2}\left\|(-\Delta)^{\frac{\sigma}{2}}|u|^{2}\right\|_{L^{2}}^{2} .
\end{aligned}
$$

We finally obtain that

$$
\mathscr{E}(u) \geq \frac{1}{2} e^{\xi^{-1}\left(\|u\|_{H^{s-}}\right)}\left(\left\||u|^{2}\right\|_{\dot{H}^{\sigma}}^{2}+\left\|\Pi^{N}|u|^{2} u\right\|_{L^{2}}^{2}+\|u\|_{H^{s}}^{2}+\left\||u|^{2}\right\|_{\dot{H}^{s-\sigma}}^{2}\right) .
$$

(b) For $0<s \leq \sigma$ : Since $\sigma$ still lies in $(0,1]$, we can use the Córdoba-Córdoba inequality to obtain

$$
\left\langle(-\Delta)^{\sigma} u,|u|^{2} u\right\rangle \geq \frac{1}{2}\left\|(-\Delta)^{\frac{\sigma}{2}}|u|^{2}\right\|_{L^{2}}^{2}
$$

However, we cannot use the Córdoba-Córdoba inequality for $(-\Delta)^{s-\sigma}$ in the term $\left\langle(-\Delta)^{s-\sigma} u,|u|^{2} u\right\rangle$ since $s \leq \sigma$. We instead employ a direct estimation (using in particular the Sobolev embedding $L^{4}=W^{0,4} \subset W^{s-\sigma, 4}$ ):

$$
\left\langle(-\Delta)^{s-\sigma} u,|u|^{2} u\right\rangle \geq-\left\|(-\Delta)^{s-\sigma} u\right\|_{L^{4}}\|u\|_{L^{4}}^{3} \geq-C\|u\|_{L^{4}}^{4} .
$$

We arrive at

$$
\mathscr{E}(u) \geq \frac{1}{2} e^{\xi^{-1}\left(\|u\|_{H^{s-}}\right)}\left(\left\||u|^{2}\right\|_{\dot{H}^{\sigma}}^{2}+\left\|\Pi^{N}|u|^{2} u\right\|_{L^{2}}^{2}+\|u\|_{H^{s}}^{2}-C\|u\|_{L^{4}}^{4}\right) .
$$

Now, let us set

$$
\mathscr{M}(u):=M^{\prime}(u, \mathscr{L}(u)),
$$


where $M(u)=\frac{1}{2}\|u\|_{L^{2}}^{2}$. We obtain that

$$
\mathscr{M}(u)=e^{\xi^{-1}\left(\|u\|_{H^{s-}}\right)}\left(\|u\|_{L^{4}}^{4}+\|u\|_{H^{s-\sigma}}^{2}\right) .
$$

This gives us that

$$
\mathscr{E}(u) \geq \frac{1}{2} e^{\xi^{-1}\left(\|u\|_{H^{s-}}\right)}\left(\left\||u|^{2}\right\|_{\dot{H}^{\sigma}}^{2}+\left\|\Pi^{N}|u|^{2} u\right\|_{L^{2}}^{2}+\|u\|_{H^{s}}^{2}\right)-\frac{C}{2} \mathscr{M}(u) .
$$

Remark 4.2 Notice that for $\sigma<\frac{1}{2}$, we do not have an estimate of $\mathscr{E}(u)$ for $s \in$ $(\sigma, d / 2)$. So we construct global solutions in that case using the arguments in Sect. 6. Surprisingly enough, we construct solutions, for such $\sigma$, in lower regularities $s \in$ $(0, \sigma)$ since in that cases the need estimates are obtained through the control of $\mathscr{E}(u)$ established above (see Sect. 6).

For any $\sigma \in(0,1]$, Assumption 5 is now fulfilled for $s \in(d / 2, \infty) \cup$ $[\max (1 / 2, \sigma), 1+\sigma] \cup[0, \sigma]$. Notice that the term $-\frac{C}{2} \mathscr{M}(u)$ in (4.8) does not have any obstruction because it is controlled under expectation (see (4.18) for details of the Itô estimate on $M(u)$ ). It's expectation is just added the to right hand side of (3.16) and similarly for absolute $\tilde{C}$ in (4.6).

In particular, we notice that

$$
\begin{aligned}
\left\|u^{2}\right\|_{\dot{H}^{\sigma}}^{2} & +\|u\|_{H^{s}}^{2}+\left\|\Pi^{N}|u|^{2} u\right\|_{L^{2}}^{2} \leq\left(\left\|u^{2}\right\|_{\dot{H}^{\sigma}}^{2}\right. \\
& \left.+\|u\|_{H^{s}}^{2}+\left\|\Pi^{N}|u|^{2} u\right\|_{L^{2}}^{2}\right) e^{\xi^{-1}\left(\|u\|_{H^{s-}}\right)} \leq 2 \mathscr{E}(u)+C \mathscr{M}(u),
\end{aligned}
$$

where $C=C(s, \sigma)$ does not depend on $(u, N, \alpha)$.

\subsection{Fulfillment of Assumption 6 (The stochastic GWP)}

For simplicity we present the proof of stochastic GWP in the case $0 \leq s \leq 1+\sigma$, we then consider the corresponding dissipation $\mathscr{L}(u)$. The other case involves a simpler dissipation operator and can be treated similarily.

We follow the well-known Da Prato-Debussche decomposition [19] (also known as Bourgain decomposition [6]). Let us consider the following linear stochastic equation

$$
d z=-\mathbf{i}(-\Delta)^{\sigma} z d t+\sqrt{\alpha} d W^{N},\left.\quad z\right|_{t=0}=0
$$

This equation admits a unique solution known as stochastic convolution:

$$
z(t)=\sqrt{\alpha} \int_{0}^{t} e^{-\mathbf{i}(t-s)(-\Delta)^{\sigma}} d W^{N}(s)
$$


It follows the Itô theory that $z(t)$ is an $\mathscr{F}_{t}$-martingale. The Doob maximal inequality combined with the Itô isometry shows that, for any $T>0$

$$
\mathbb{E} \sup _{t \in[0, T]}\|z(t)\|_{L^{2}} \leq C_{T} \sqrt{\alpha} .
$$

We notice that $z$ exists globally in $t$ for almost all $\omega \in \Omega$. Let $u_{0}$ be $E^{N}$-valued $\mathscr{F}_{0}-$ independent random variable. Fix $\omega$ such $z^{\omega}$ exists globally in time, and consider the nonlinear deterministic equation

$$
\partial_{t} v=F(v, z), \quad v(t=0)=u_{0}^{\omega},
$$

where $F(v, z)=-\mathbf{i}\left((-\Delta)^{\sigma} v+|v+z|^{2}(v+z)\right)-\alpha e^{\xi^{-1}\left(\|v+z\|_{H^{s-}}\right)}\left[\Pi^{N}|v+z|^{2}(v+\right.$ $\left.z)+(-\Delta)^{s-\sigma}(v+z)\right]$.

We remark that for a global solution $v$ to (4.12), we have that $u=v+z$ is a global solution to (4.5) supplemented with the initial condition $u(t=0)=u_{0}^{\omega}$.

The local existence for (4.12) follows from the classical Cauchy-Lipschitz theorem since $F(v, z)$ is smooth. Let us show that this local solution is in fact global. By an standard iteration argument, it suffices to show that the $L^{2}$-norm does not blow up in finite time. We have that

$$
\begin{aligned}
\partial_{t}\|v\|_{L^{2}}^{2}= & -2 \mathbf{i}\left\langle v, \Pi^{N}|v+z|^{2}(v+z)\right\rangle-2 \alpha e^{\xi^{-1}\left(\|v+z\|_{H^{s-}}\right)}\left[\left\langle v, \Pi^{N}|v+z|^{2}(v+z)\right\rangle\right. \\
& \left.+\left\langle v,(-\Delta)^{s-\sigma}(v+z)\right\rangle\right] .
\end{aligned}
$$

By adding $z-z$ and using Cauchy-Schwarz, we obtain

$$
\partial_{t}\|v\|_{L^{2}}^{2} \leq \frac{C_{N}\|z\|_{L^{2}}^{4}}{\alpha}+e^{\xi^{-1}\left(\|v+z\|_{H^{s-}}\right)}\left(-\|v\|_{H^{s-\sigma}}^{2}+\tilde{C}_{N}\left(\|z\|_{L^{2}}^{2}+\|z\|_{L^{4}}^{4}\right)\right) .
$$

We then have two complementary cases for a $t \in[0, T]$ :

(1) either $\|v\|_{H^{s-\sigma}}^{2} \leq \tilde{C}_{N}\left(\|z\|_{L^{2}}^{2}+\|z\|_{L^{4}}^{4}\right)$; in this case

$$
\partial_{t}\|v\|_{L^{2}}^{2} \leq C_{\alpha}^{0}(\omega, T)
$$

(2) or $\|v\|_{H^{s-\sigma}}^{2}>\tilde{C}_{N}\left(\|z\|_{L^{2}}^{2}+\|z\|_{L^{4}}^{4}\right)$; in this case

$$
e^{\xi^{-1}\left(\|v+z\|_{H^{s-}}\right)}\left(-\|v\|_{H^{s-\sigma}}^{2}+\tilde{C}_{N}\|z\|_{L^{2}}^{2}\right) \leq 0,
$$

and

$$
\partial_{t}\|v\|_{L^{2}}^{2} \leq C_{\alpha}^{1}(\omega, T)
$$

Taking $C_{\alpha}=\max \left(C_{\alpha}^{0}, C_{\alpha}^{1}\right)$, we obtain the wished finiteness of $\|v\|_{L^{2}}$. 
Now, using the mean value theorem and the Gronwall lemma, we find for $u$ and $v$ two solutions to (4.5) that

$$
\|u(t)-v(t)\|_{L^{2}} \leq\|u(0)-v(0)\|_{L^{2}} e^{\sup _{x \in D} \int_{0}^{1}\left|f^{\prime}(r u+(1-r) v)\right| d r},
$$

where $f(u)=F(u, 0)$.

Standard arguments show that the constructed solution $u$ is adapted to $\sigma\left(u_{0}, \mathscr{F}_{t}\right)$.

\subsection{Fulfillment of Assumption 7 (Zero viscosity limit, $\alpha \rightarrow 0$ )}

The difficulty in the convergence of Assumption 7 is the fact that both $P_{t, \alpha_{k}}^{N *}$ and $\mu_{\alpha_{k}}^{N}$ depend on $\alpha_{k}$, we then need some uniformity in the convergence $u_{\alpha_{k}}^{N}(t, \cdot) \rightarrow \phi_{t}^{N}(\cdot)$ as $\alpha_{k} \rightarrow 0$. The following lemma gives the needed uniform convergence. To simplify the notation, we use the abuse of notation: $P_{t, \alpha_{k}}^{N *}=: P_{t, k}^{N *}, \mu_{\alpha_{k}}^{N}=: \mu_{k}^{N}$ and $u_{\alpha_{k}}=: u_{k}$.

Lemma 4.3 Let $T>0$. For any $R>0$, any $r>0$,

$$
\sup _{u_{0} \in B_{R}\left(L^{2}\right)} \sup _{t \in[0, T]} \mathbb{E}\left(\left\|u_{k}\left(t, \Pi^{N} u_{0}\right)-\phi_{t}^{N} \Pi^{N} u_{0}\right\|_{L^{2}} 1_{S_{r, k}(t)}\right) \rightarrow 0, \quad \text { as } k \rightarrow \infty,
$$

where

$$
S_{r, k}(t)=\left\{\omega \in \Omega \mid \max \left(\left\|z_{\alpha_{k}}^{\omega}(t)\right\|_{L^{2}}, \sqrt{\alpha_{k}}\left|\sum_{m=1}^{N} \int_{0}^{t} a_{m}\left\langle u, e_{m}\right\rangle d \beta_{s}^{\omega}\right|\right) \leq r\right\} .
$$

We postpone the proof of Lemma 4.3. We remark that, using the Ito isometry and the Chebyshev inequality we have

$$
\mathbb{E}\left(1-1_{S_{r, k}}(t)\right) \leq \frac{C t}{r^{2}}
$$

where $C$ does not depend on $(r, k, t)$.

Let $f: L^{2} \rightarrow \mathbb{R}$ be a bounded Lipschitz function. Without any loss of generality we can assume that $f$ is bounded by 1 and its Lipschitz constant is also 1 . We have

$$
\begin{aligned}
\left\langle P_{t, k}^{N *} \mu_{k}^{N}, f\right\rangle-\left\langle P_{t}^{N *} \mu^{N}, f\right\rangle & =\left\langle\mu_{k}^{N}, P_{t, k}^{N} f\right\rangle-\left\langle\mu^{N}, P_{t}^{N} f\right\rangle \\
& =\left\langle\mu_{k}^{N},\left(P_{t, k}^{N}-P_{t}^{N}\right) f\right\rangle-\left\langle\mu^{N}-\mu_{k}^{N}, P_{t}^{N} f\right\rangle \\
& =A-B .
\end{aligned}
$$

We see that $B \rightarrow 0$ as $k \rightarrow \infty$ according to the weak convergence $\mu_{k}^{N} \rightarrow \mu^{N}$. Using the boundedness of $f$ we obtain

$$
|A| \leq \int_{B_{R}\left(L^{2}\right)}\left|P_{t, k}^{N} f(w)-P_{t}^{N} f(w)\right| \mu_{k}^{N}(d w)+2 \mu_{k}^{N}\left(L^{2} \backslash B_{R}\left(L^{2}\right)\right)=: A_{1}+A_{2} .
$$


Now from (3.16),

$$
\int_{L^{2}}\|u\|_{L^{2}}^{2} \mu_{k}^{N}(d u) \leq \int_{L^{2}} \mathscr{E}(u) \mu_{k}^{N}(d u) \leq C,
$$

where $C$ does not depend on $(N, \alpha)$. Combining this with the Chebyshev inequality we obtain that

$$
A_{2} \leq \frac{2 C}{R^{2}}
$$

Using the boundedness and Lipschitz properties of $f$, we obtain

$$
A_{1} \leq A_{1,1}+A_{1,2}
$$

where

$$
\begin{aligned}
& A_{1,1}=\int_{B_{R}\left(L^{2}\right)}\left\|\mathbb{E} u_{k}^{N}\left(t, \Pi^{N} w\right)-\phi_{t} w\right\|_{L^{2}} 1_{S_{r}} \mu_{k}^{N}(d w) \\
& A_{1,2}=2 \int_{L^{2} \backslash B_{R}\left(L^{2}\right)} \mathbb{E}\left(1-1_{S_{r}}\right) \mu_{k}^{N}(d w) .
\end{aligned}
$$

Using the (4.14) we obtain that

$$
A_{1,2} \leq \frac{2 C t}{r^{2}}
$$

We finally obtain

$$
|A| \leq A_{1,1}+\frac{2 C t}{r^{2}}+\frac{2 C}{R^{2}} .
$$

We pass to the limit on $k$ first, by applying apply Lemma 4.3 , we obtain $A_{1,1} \rightarrow 0$. Then we take the limits $r \rightarrow \infty$ and $R \rightarrow \infty$, we arrive at the conclusion of Assumption 7 .

Now let us prove Lemma (4.3):

Proof of Lemma 4.3 Let $u_{0} \in B_{R}\left(L^{2}\right)$ and $u_{k}$ and $u$ be the solutions of (4.5) (with viscosity $\alpha_{k}$ ) and (4.1) starting at $u_{0}$, respectively. Recall that $u_{k}$ can be decomposed as $u_{k}=v_{k}+z_{k}$ where $v_{k}$ is the solution of (4.12) with initial datum $u_{0}$ and $z_{k}$ is given by (4.10) with $\alpha_{k}$. In order to prove Lemma 4.3, it suffice to show that

$$
\sup _{u_{0} \in B_{R}} \sup _{t \in[0, T]} \mathbb{E}\left(\left\|v_{k}-u\right\|_{L^{2}} 1_{S_{r, k}(t)}\right) \rightarrow 0, \quad \text { as } k \rightarrow \infty
$$

Indeed, we already have by (4.11) that $\mathbb{E} \sup _{t \in[0, T]}\left\|z_{k}(t)\right\|_{L^{2}} \rightarrow 0$ as $k \rightarrow \infty$. Set $w_{k}=v_{k}-u$. We will treat the case $s \leq 1+\sigma$ which is more delicate. We then consider the equation satisfied by $w_{k}$ :

$$
\partial_{t} w_{k}=\mathbf{i} \Delta w_{k}+\Pi^{N}\left[w_{k} f\left(v_{k}, u, z_{k}\right)+z_{k} g\left(v_{k}, z_{k}\right)\right]
$$




$$
-\alpha_{k} e^{\xi^{-1}\left(\left\|v_{k}+z_{k}\right\|_{H^{s-}}\right)}(-\Delta)^{s-\sigma}\left(v_{k}+z_{k}\right)
$$

where $f$ and $g$ is a homogeneous of degree 2 complex polynomial. We claim that $\lim _{k \rightarrow \infty}\left\|w_{k}\right\|_{L^{2}}=0$ almost surely. Indeed, by taking the dot product with $w_{k}$, we obtain after the use of the Gronwall inequality,

$$
\begin{aligned}
& \sup _{t \in[0, T]}\left\|w_{k}\right\|_{L^{2}}^{2} \leq e^{T\left(1+z_{N}^{2}+\left\|f\left(v_{k}, z_{k}\right)\right\|_{L^{\infty}}\right)}\left(\int_{0}^{T}\|z\|_{L^{2}}^{2} d t+\alpha_{k} T\right)
\end{aligned}
$$

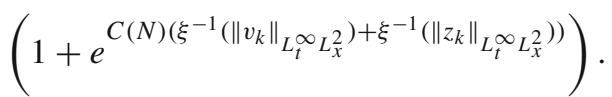

We pass to the limit $k \rightarrow \infty$ with the use of (4.11) to obtain the claim.

Now, writing the Itô formula for $\left\|u_{k}\right\|_{L^{2}}^{2}$, we have

$$
\begin{aligned}
\left\|u_{k}\right\|_{L^{2}}^{2}+2 \alpha_{k} \int_{0}^{t} \mathscr{M}\left(u_{k}\right) d \tau= & \left\|\Pi^{N} u_{0}\right\|_{L^{2}}^{2}+\alpha_{k} \frac{A_{0}^{N}}{2} t \\
& +2 \sqrt{\alpha_{k}} \sum_{m \leq N} a_{m} \int_{0}^{t}\left\langle u_{k}, e_{m}\right\rangle d \beta_{m}
\end{aligned}
$$

where

$$
\mathscr{M}(u)=M^{\prime}(u, \mathscr{L}(u)) \geq 0 .
$$

Since $\alpha_{k} \leq 1$, we have that, on the set $S_{r, k}(t)$,

$$
\left\|u_{k}\right\|_{L^{2}}^{2} \leq\left\|\Pi^{N} u_{0}\right\|_{L^{2}}^{2}+C(r, N) t
$$

where $C(r, N)$ does not depend on $k$. Hence we see that, on $S_{r, k}(t)$,

$$
\left\|w_{k}\right\|_{L^{2}} \leq\left\|v_{k}\right\|_{L^{2}}+\left\|z_{k}\right\|_{L^{2}} \leq\left\|u_{k}\right\|_{L^{2}}+2\left\|z_{k}\right\|_{L^{2}} \leq\left\|u_{0}\right\|_{L^{2}}+3 C(r, N) t .
$$

In particular, we have the following two estimates:

$$
\sup _{k \geq 1} \sup _{u_{0} \in B_{R}}\left\|w_{k}\right\|_{L_{t}^{\infty} L_{x}^{2}} \mathbb{1}_{S_{r, k}} \leq R+3 C(r, N) T
$$

Hence coming back to (4.15) and using the (deterministic) conservation $\|u(t)\|_{L^{2}}=$ $\left\|P_{N} u_{0}\right\|_{L^{2}}$ and the estimate (4.16), we obtain

$$
\sup _{u_{0} \in B_{R}}\left\|w_{k}\right\|_{L_{t}^{\infty} L_{x}^{2}}^{2} \mathbb{1}_{S_{r}} \leq A(R, N, r, T)\left(\alpha_{k}+\left\|z_{k}\right\|_{L_{t}^{1} L_{x}^{2}}\right)
$$

Therefore, using again the bound (4.11), we obtain the almost sure convergence $\left\|z_{k}\right\|_{L^{2}} \rightarrow 0$ (as $k \rightarrow \infty$, up to a subsequence), we obtain then the almost sure 
convergence

$$
\lim _{k \rightarrow \infty} \sup _{u_{0} \in B_{R}}\left\|w_{k}\right\|_{L_{t}^{\infty} L_{x}^{2}}^{2} \mathbb{1}_{S_{r}}=0
$$

Now, we use (4.16) and the Lebesgue dominated convergence theorem to obtain

$$
\mathbb{E} \sup _{u_{0} \in B_{R}}\left\|w_{k}\right\|_{L_{t}^{\infty} L_{x}^{2}} \mathbb{1}_{S_{r}} \rightarrow 0, \text { as } k \rightarrow \infty
$$

The proof of Lemma 4.3 is finished.

\subsection{Estimates}

Let us recall that

$$
\mathscr{M}(u):=M^{\prime}(u, \mathscr{L}(u))=\langle u, \mathscr{L}(u)\rangle .
$$

Proposition 4.4 We have that

$$
\int_{L^{2}} \mathscr{M}(u) \mu(d u)=\frac{A_{0}}{2} .
$$

Proof of Proposition 4.4 We present the more difficult case in which the dissipation is given by

$$
\mathscr{L}(u)=e^{\xi^{-1}\left(\|u\|_{H^{s-}}\right)}\left(\Pi^{N}|u|^{2} u+(-\Delta)^{s-\sigma} u\right), \quad \text { where } 0 \leq s \leq 1+\sigma .
$$

In this case we have

$$
\mathscr{M}(u)=e^{\xi^{-1}\left(\|u\|_{H^{s-}}\right)}\left(\|u\|_{L^{4}}^{4}+\|u\|_{H^{s-\sigma}}^{2}\right) .
$$

The other case can be proved using a similar procedure. We split the proof in different steps:

(1) Step 1: Identity for the $\left(\mu_{\alpha}^{N}\right)$.

Applying the Itô formula to $M(u)$, where $u$ is the solution to (4.5), we obtain

$$
d M(u)=\left[-\alpha \mathscr{M}(u) d t+\frac{\alpha}{2} A_{0}^{N}\right] d t+\sqrt{\alpha} \sum_{m=0}^{N} a_{m}\left\langle u, e_{m}\right\rangle d \beta_{m}
$$

Integrating in $t$ and in ensemble with repect to $\mu_{\alpha}^{N}$, we obtain

$$
\int_{L^{2}} \mathscr{M}(u) \mu_{\alpha}^{N}(d u)=\frac{A_{0}^{N}}{2},
$$


this identity used the invariance $\mu_{\alpha}^{N}$.

Let us now establish an auxiliary bound. Apply the Itô formula to $M^{2}(u)$

$$
\begin{aligned}
d M^{2}(u)= & {\left[\alpha M(u)\left(-\mathscr{M}(u)+\frac{A_{0}^{N}}{2}\right)+\frac{\alpha}{2} \sum_{m \leq N}\left\langle u, e_{m}\right\rangle^{2}\right] d t } \\
& +2 \sqrt{\alpha} M(u) \sum_{m \leq N}\left\langle u, e_{m}\right\rangle d \beta_{m} .
\end{aligned}
$$

Integrating in $t$ and with respect respect to $\mu_{\alpha}^{N}$ we arrive at

$$
\int_{L^{2}} M(u) \mathscr{M}(u) \mu_{\alpha}^{N}(d u) \leq C,
$$

where $C$ does not depend in $(N, \alpha)$. The estimate above is obtained after the following remark

$$
\int_{L^{2}} M(u) \mu_{\alpha}^{N}(d u) \leq \int_{L^{2}} \mathscr{E}(u) \mu_{\alpha}^{N}(d u) \leq C_{1}
$$

where $C_{1}$ is independent of $(N, \alpha)$.

(2) Step 2: Identity for the $\left(\mu^{N}\right)$. By usual arguments, we obtain the following estimates for $\left(\mu^{N}\right)$ :

$$
\begin{gathered}
\int_{L^{2}} \mathscr{M}(u) \mu^{N}(d u)=\frac{A_{0}^{N}}{2} ; \\
\int_{L^{2}} M(u) \mathscr{M}(u) \mu^{N}(d u) \leq C .
\end{gathered}
$$

We do not give details of proof of the identities. In the next step we will prove more delicate estimates whose proof is highly more difficult as the passage to the limit cannot use any finite-dimensional advantage.

(3) Step 3: Identity for the $\mu$. In this part of the proof we perform the passage to the limit $N \rightarrow \infty$ in (4.20) in order to obtain the identity (4.17).

The inequality

$$
\int_{L^{2}} \mathscr{M}(u) \mu(d u) \leq \frac{A_{0}}{2}
$$

can be obtained by invoking lower semi-continuity of $\mathscr{M}$. The other way around, the analysis is more challenging. In [54] a similar identity was established with the use of an auxiliary estimate on a quantity of type $E(u) \mathscr{E}(u)$. But in our context, such an estimate is not available. Indeed, in order to obtain it, we shall apply the Itô formula on $E^{2}$, the dissipation will be $E(u) \mathscr{E}(u)$; but other terms including $A_{\sigma}^{N} E(u)$ have to be controlled in expectation. However, we do not have an $N$-independent control on $\int_{L^{2}} E(u) \mu^{N}(d u)$ because this term is smoother than 
$\mathscr{E}(u)$ for $s<\sigma$. Therefore the latter cannot be exploited. We remark, on the other hand, that this is why the measure $\mu$ is expected to be concentrated on regularities lower than the energy space $H^{\sigma}$. Now, without a control on $E(u) \mathscr{E}(u)$, we can only handle the weaker quantity $M(u) \mathscr{M}(u)$ from (4.19). So our proof here will be more tricky than that in [54].

Coming back to the proof of the remaining inequality, we write for some fixed frequency $F$, the frequency decomposition (setting $\Pi^{>F}=1-\Pi^{F}$ )

$$
\begin{aligned}
\frac{A_{0}^{N}}{2} \leq & \mathbb{E} e^{\xi^{-1}\left(\left\|u^{N}\right\|_{H^{s-}}\right)}\left(\left\|\Pi^{F}\left|u^{N}\right|^{2}\right\|_{L^{2}}^{2}+\left\|\Pi^{F} u^{N}\right\|_{H^{s-\sigma}}^{2}\right) \\
& +\mathbb{E} e^{\xi^{-1}\left(\left\|u^{N}\right\|_{H^{s-}}\right)}\left(\left\|\Pi^{>F}\left|u^{N}\right|^{2}\right\|_{L^{2}}^{2}+\left\|\Pi^{>F} u^{N}\right\|_{H^{s-\sigma}}^{2}\right) \\
= & : i+i i,
\end{aligned}
$$

where $u^{N}$ is distributed by $\mu^{N}$. Let us follow the following sub-steps:

(a) First, $i$ can be estimated by using the control on $\mathscr{E}(4.7)$ as follows

$$
\begin{aligned}
i i & \lesssim F^{-\sigma}\left(\mathbb{E} e^{\xi^{-1}\left(\left\|u^{N}\right\|_{H^{s-}}\right)}\left(\left\|\Pi^{>F}\left|u^{N}\right|^{2}\right\|_{H^{\sigma}}^{2}+\left\|u^{N}\right\|_{\dot{H}^{s}}^{2}\right)\right) \\
& \lesssim F^{-\sigma} \mathbb{E} \mathscr{E}\left(u^{N}\right) \lesssim F^{-\sigma} C .
\end{aligned}
$$

(b) As for $i$, we can split it by using localization in $H^{s-}$ (notice that by using the Skorokhod theorem $\left(u^{N}\right)$ converges almost surely on $H^{s-}$ to some $H^{s-}$-valued random variable $u$ ). For any fixed $R>0$ we set $\chi_{R}=$ $1_{\left\{\left\|u^{N}\right\|_{H^{s-}} \geq R\right\}}$. We have

$$
\begin{aligned}
& \mathbb{E} e^{\xi^{-1}\left(\left\|u^{N}\right\|_{H^{s-}}\right)}\left(\left\|\Pi^{F}\left|u^{N}\right|^{2}\right\|_{L^{2}}^{2}+\left\|\Pi^{F} u^{N}\right\|_{H^{s-\sigma}}^{2}\right) \chi_{R} \\
& \lesssim \mathbb{E} e^{\xi^{-1}\left(\left\|u^{N}\right\|_{H^{s-}}\right)}\left(\left\|\Pi^{F}\left|u^{N}\right|^{2}\right\|_{H^{\frac{d}{2}}}^{2}+\left\|\Pi^{F} u^{N}\right\|_{H^{s-\sigma}}^{2}\right) \chi_{R} \\
& \lesssim \mathbb{E} e^{\xi^{-1}\left(\left\|u^{N}\right\|_{H^{s-}}\right)}\left(\left\|u^{N}\right\|_{H^{\frac{d}{2}+}}^{4}+\left\|\Pi^{F} u^{N}\right\|_{H^{s-\sigma}}^{2}\right) \chi_{R} \\
& \lesssim F^{\frac{d}{2}+} \mathbb{E} e^{\xi^{-1}\left(\left\|u^{N}\right\|_{H^{s-}}\right)}\left(\left\|u^{N}\right\|_{L^{2}}^{4}+\left\|u^{N}\right\|_{H^{s-\sigma}}^{2}\right) \chi_{R} \\
& \lesssim F^{\max \left(\frac{d}{2}+, s-\sigma\right)} R^{-2} \mathbb{E} e^{\xi^{-1}\left(\left\|u^{N}\right\|_{H^{s-}}\right)}\left\|u^{N}\right\|_{H^{s-}}^{2}\left(\left\|u^{N}\right\|_{L^{2}}^{4}+\left\|u^{N}\right\|_{H^{s-\sigma}}^{2}\right) \\
& \lesssim F^{\max \left(\frac{d}{2}+, s-\sigma\right)+s} R^{-2} \mathbb{E}\left\|u^{N}\right\|_{L^{2}}^{2} e^{\xi^{-1}\left(\left\|u^{N}\right\|_{H^{s-}}\right)}\left[\left\|u^{N}\right\|_{L^{4}}^{4}+\left\|u^{N}\right\|_{H^{s-\sigma}}^{2}\right] .
\end{aligned}
$$

Let us use the estimate (4.20) and (4.21) to arrive at

$$
\begin{aligned}
& \mathbb{E} e^{\xi^{-1}\left(\left\|u^{N}\right\|_{H^{s-}}\right)}\left(\left\|\Pi^{F}\left|u^{N}\right|^{2}\right\|_{L^{2}}^{2}+\left\|\Pi^{F} u^{N}\right\|_{H^{s-\sigma}}^{2}\right) \chi_{R} \\
& \lesssim F^{\max \left(\frac{d}{2}+, s-\sigma\right)+s} R^{-2} \mathbb{E} M\left(u^{N}\right) \mathscr{M}\left(u^{N}\right) \\
& \lesssim F^{\max \left(\frac{d}{2}+, s-\sigma\right)+s} R^{-2} C,
\end{aligned}
$$

where $C$ does not depend on $N$. 
(c) On the other hand, we have the following convergence, thanks to the Lebesgue dominated convergence theorem,

$$
\begin{aligned}
& \lim _{N \rightarrow \infty} \mathbb{E} e^{\xi^{-1}\left(\left\|u^{N}\right\|_{H^{s-}}\right)}\left(\left\|\Pi^{F}\left|u^{N}\right|^{2}\right\|_{L^{2}}^{2}+\left\|\Pi^{F} u^{N}\right\|_{H^{s-\sigma}}^{2}\right)\left(1-\chi_{R}\right) \\
& =\mathbb{E} e^{\xi^{-1}\left(\|u\|_{H^{s-}}\right)}\left(\left\|\Pi^{F}|u|^{2}\right\|_{L^{2}}^{2}+\left\|\Pi^{F} u\right\|_{H^{s-\sigma}}^{2}\right)\left(1-\chi_{R}\right) .
\end{aligned}
$$

Gathering all this, we obtain, after the limit $N \rightarrow \infty$, that

$$
\begin{aligned}
\frac{A_{0}}{2} \leq & \mathbb{E} e^{\xi^{-1}\left(\|u\|_{H^{s-}}\right)}\left(\left\|\Pi^{F}|u|^{2}\right\|_{L^{2}}^{2}+\left\|\Pi^{F} u\right\|_{H^{s-\sigma}}^{2}\right)\left(1-\chi_{R}\right)+F^{-\sigma} C_{1} \\
& +F^{\max \left(\frac{d}{2}+, s-\sigma\right)+s} R^{-2} C_{2} .
\end{aligned}
$$

We let $R \rightarrow \infty$, then $F \rightarrow \infty$ and obtain

$$
\frac{A_{0}}{2} \leq e^{\xi^{-1}\left(\|u\|_{H^{s-}}\right)} \mathbb{E}\left(\|u\|_{L^{4}}^{4}+\|u\|_{H^{s-\sigma}}^{2}\right)
$$

The proof of Proposition 4.4 is finished.

Remark 4.5 The identity (4.17) is crucial for establishing the non-degeneracy properties of the measure $\mu$. It trivially rule out the Dirac measure at 0 , notice that the Dirac at 0 is a trivial invariant measure for FNLS.

Also, by considering a noise $\kappa d W,(4.17)$ becomes

$$
\mathbb{E} \mathscr{M}(u)=\frac{\kappa A_{0}}{2}
$$

Such scaled noises provide invariant measures $\mu_{\kappa}$ for FNLS, all satisfying (4.22). Define a cumulative measure $\mu^{*}$ by a convex combination

$$
\mu^{*}=\sum_{j=0}^{\infty} \rho_{j} \mu_{\kappa_{j}}
$$

where $\kappa_{j} \uparrow \infty$ and $\sum_{0}^{\infty} \rho_{j}=1$. The measure $\mu^{*}$ is invariant for FNLS. Moreover, for any $K>0$, we can find a positive $\mu^{*}$-measure set of data whose $H^{s}$-norms are larger than $K$.

In order to finish the proof of Theorem 1.1, we present in the section below the fulfillment of Assumptions 1 and 2.

\section{End of the proof of Theorem 1.1: Local well-posedness}

In this section, we present a deterministic local well-posedness result for $\sigma \in\left[\frac{1}{2}, 1\right]$ in (1.1), which heavily replies on a bilinear Strichartz estimate obtained in Sect. 5.1. 
See also Sect. 3 in [55]. We also show a convergence from Galerkin approximations of FNLS to FNLS.

Theorem 5.1 (Deterministic local well-posedness on the unit ball) The fractional NLS (1.1) with $\sigma \in\left[\frac{1}{2}, 1\right]$ is locally well-posed for radial data $u_{0} \in H_{\text {rad }}^{s}\left(B^{d}\right), s>s_{l}(\sigma)$, where $s_{l}(\sigma)$ is defined as in (1.3). More precisely, let us first fix $s>s_{l}(\sigma)$ (defined as in (1.3)), and for every $R>0$, we set $\delta=\delta(R)=c R^{-2 s}$ for some $c \in(0,1]$. Then there exists $b>\frac{1}{2}$ and $C, \widetilde{C}>0$ such that every $u_{0} \in H_{\text {rad }}^{s}\left(B^{d}\right)$ satisfying $\left\|u_{0}\right\|_{H_{r a d}^{s}\left(B^{d}\right)} \leq R$, there exists a unique solution of $(1.1)$ in $X_{\sigma, r a d}^{s, b}\left([-\delta, \delta] \times B^{d}\right)$ with initial condition $u(0)=u_{0}$. Moreover,

$$
\|u\|_{L_{t}^{\infty} H_{x}^{s}\left([-\delta, \delta] \times B^{d}\right)} \leq C\|u\|_{X_{\sigma, r a d}^{s, b}\left([-\delta, \delta] \times B^{d}\right)} \leq \widetilde{C}\left\|u_{0}\right\|_{H_{\text {rad }}^{s}\left(B^{d}\right)} .
$$

Remark 5.2 The function $f$ in Assumption 1 is found to be equal to $\delta(x)=c x^{-2 s}$.

\subsection{Bilinear Strichartz estimates}

In this subsection, we prove the bilinear estimates that will be used in the rest of this section. The proof is adapted from [1] with two dimensional modification and a different counting lemma.

Lemma 5.3 (Bilinear estimates for fractional NLS) For $\sigma \in\left[\frac{1}{2}, 1\right], j=1,2, N_{j}>0$ and $u_{j} \in L_{\text {rad }}^{2}\left(B^{d}\right)$ satisfying

$$
\mathbf{1}_{\sqrt{-\Delta} \in\left[N_{j}, 2 N_{j}\right]} u_{j}=u_{j},
$$

we have the following bilinear estimates.

(1) The bilinear estimate without derivatives.

Without loss of generality, we assume $N_{1} \geq N_{2}$, then for any $\varepsilon>0$

$$
\left\|S_{\sigma}(t) u_{1} S_{\sigma}(t) u_{2}\right\|_{L_{t, x}^{2}\left((0,1) \times B^{d}\right)} \lesssim N_{2}^{\frac{d-1}{2}+\varepsilon}\left\|u_{1}\right\|_{L_{x}^{2}\left(B^{d}\right)}\left\|u_{2}\right\|_{L_{x}^{2}\left(B^{d}\right)} .
$$

(2) The bilinear estimate with derivatives.

Moreover, if $u_{j} \in H_{0}^{1}\left(B^{d}\right)$, then for any $\varepsilon>0$

$$
\left\|\nabla S_{\sigma}(t) u_{1} S_{\sigma}(t) u_{2}\right\|_{L_{t, x}^{2}\left((0,1) \times B^{d}\right)} \lesssim N_{1} N_{2}^{\frac{d-1}{2}+\varepsilon}\left\|u_{1}\right\|_{L_{x}^{2}\left(B^{d}\right)}\left\|u_{2}\right\|_{L_{x}^{2}\left(B^{d}\right)} .
$$

Remark 5.4 Notice that in (5.1) and (5.2), the upper bounds are independent on the fractional power $\sigma$. This is because a counting estimate in Claim 5.7 does not see difference on $\sigma$. Hence the local well-posedness index is uniform for $\sigma \in\left[\frac{1}{2}, 1\right)$.

Lemma 5.5 (Bilinear estimates for classical NLS) Under the same setup as in Lemma 5.3, the bilinear estimate analogue is given by

$$
\left\|S_{1}(t) u_{1} S_{1}(t) u_{2}\right\|_{L_{t, x}^{2}\left((0,1) \times B^{d}\right)} \lesssim N_{2}^{\frac{d-2}{2}+\varepsilon}\left\|u_{1}\right\|_{L_{x}^{2}\left(B^{d}\right)}\left\|u_{2}\right\|_{L_{x}^{2}\left(B^{d}\right)}
$$


$\left\|\nabla S_{1}(t) u_{1} S_{1}(t) u_{2}\right\|_{L_{t, x}^{2}\left((0,1) \times B^{d}\right)} \lesssim N_{1} N_{2}^{\frac{d-2}{2}+\varepsilon}\left\|u_{1}\right\|_{L_{x}^{2}\left(B^{d}\right)}\left\|u_{2}\right\|_{L_{x}^{2}\left(B^{d}\right)}$.

Notice that the proof of Lemma 5.5 in fact can be extended from [1], hence we will only focus on the proof of Lemma 5.3 and the proof of the local theory for fractional NLS in the rest of this section.

Proposition 5.6 (Lemma 2.3 in [10]: Transfer principle) For any $b>\frac{1}{2}$ and for $j=$ $1,2, N_{j}>0$ and $f_{j} \in X_{\sigma}^{0, b}\left(\mathbb{R} \times B^{d}\right)$ satisfying

$$
\mathbf{1}_{\sqrt{-\Delta} \in\left[N_{j}, 2 N_{j}\right]} f_{j}=f_{j},
$$

one has the following bilinear estimates.

(1) The bilinear estimate without derivatives.

Without loss of generality, we assume $N_{1} \geq N_{2}$, then for any $\varepsilon>0$

$$
\left\|f_{1} f_{2}\right\|_{L_{t, x}^{2}\left(\mathbb{R} \times B^{d}\right)} \lesssim N_{2}^{\frac{d-1}{2}+\varepsilon}\left\|f_{1}\right\|_{X_{\sigma}^{0, b}\left(\mathbb{R} \times B^{d}\right)}\left\|f_{2}\right\|_{X_{\sigma}^{0, b}\left(\mathbb{R} \times B^{d}\right)} .
$$

(2) The bilinear estimate with derivatives.

Moreover, if $f_{j} \in H_{0}^{1}\left(B^{d}\right)$, then for any $\varepsilon>0$

$$
\left\|\nabla f_{1} f_{2}\right\|_{L_{t, x}^{2}\left(\mathbb{R} \times B^{d}\right)} \lesssim N_{1} N_{2}^{\frac{d-1}{2}+\varepsilon}\left\|f_{1}\right\|_{X_{\sigma}^{0, b}\left(\mathbb{R} \times B^{d}\right)}\left\|f_{2}\right\|_{X_{\sigma}^{0, b}\left(\mathbb{R} \times B^{d}\right)}
$$

Proof of Lemma 5.3 First we write

$$
u_{1}=\sum_{n_{1} \sim N_{1}} c_{n_{1}} e_{n_{1}}(r), \quad u_{2}=\sum_{n_{2} \sim N_{2}} d_{n_{2}} e_{n_{2}}(r)
$$

where $c_{n_{1}}=\left(u_{1}, e_{n_{1}}\right)_{L^{2}}$ and $d_{n_{2}}=\left(u_{2}, e_{n_{2}}\right)_{L^{2}}$. Then

$$
S_{\sigma}(t) u_{1}=\sum_{n_{1} \sim N_{1}} e^{-\mathbf{i} t z_{n_{1}}^{2 \sigma}} c_{n_{1}} e_{n_{1}}(r), \quad S_{\sigma}(t) u_{2}=\sum_{n_{2} \sim N_{2}} e^{-\mathbf{i} t z_{n_{2}}^{2 \sigma}} d_{n_{2}} e_{n_{2}}(r)
$$

Therefore, the bilinear objects that one needs to estimate are the $L_{t, x}^{2}$ norms of

$$
\begin{aligned}
& E_{0}\left(N_{1}, N_{2}\right)=\sum_{n_{1} \sim N_{1}} \sum_{n_{2} \sim N_{2}} e^{-\mathbf{i} t\left(z_{n_{1}}^{2 \sigma}+z_{n_{2}}^{2 \sigma}\right)}\left(c_{n_{1}} d_{n_{2}}\right)\left(e_{n_{1}} e_{n_{2}}\right), \\
& E_{1}\left(N_{1}, N_{2}\right)=\sum_{n_{1} \sim N_{1}} \sum_{n_{2} \sim N_{2}} e^{-\mathbf{i} t\left(z_{n_{1}}^{2 \sigma}+z_{n_{2}}^{2 \sigma}\right)}\left(c_{n_{1}} d_{n_{2}}\right)\left(\nabla e_{n_{1}} e_{n_{2}}\right) .
\end{aligned}
$$

Let us focus on (5.1) first.

$$
(\operatorname{LHS} \text { of }(5.15 .1))^{2}=\left\|E_{0}\left(N_{1}, N_{2}\right)\right\|_{L^{2}\left((0,1) \times B^{d}\right)}^{2}
$$




$$
=\int_{\mathbb{R} \times B^{d}}\left|\sum_{n_{1} \sim N_{1}} \sum_{n_{2} \sim N_{2}} e^{-\mathbf{i} t\left(z_{n_{1}}^{2 \sigma}+z_{n_{2}}^{2 \sigma}\right)}\left(c_{n_{1}} d_{n_{2}}\right)\left(e_{n_{1}} e_{n_{2}}\right)\right|^{2} d x d t
$$

Here we employ a similar argument used in the proof of Lemma 2.6 in [50]. We fix $\eta \in C_{0}^{\infty}((0,1))$, such that $\left.\eta\right|_{I} \equiv 1$ where $I$ is a slight enlargement of $(0,1)$. Thus we continue from (5.5)

$$
\begin{aligned}
(5.5) & \leq \int_{\mathbb{R} \times B^{d}} \eta(t)\left|\sum_{n_{1} \sim N_{1}} \sum_{n_{2} \sim N_{2}} e^{-\mathbf{i} t\left(z_{n_{1}}^{2 \sigma}+z_{n_{2}}^{2 \sigma}\right)}\left(c_{n_{1}} d_{n_{2}}\right)\left(e_{n_{1}} e_{n_{2}}\right)\right|^{2} d x d t \\
& =\int_{\mathbb{R} \times B^{d}} \eta(t)\left|\sum_{\tau} \sum_{\left(n_{1}, n_{2}\right) \in \Lambda_{N_{1}, N_{2}, \tau}} e^{-\mathbf{i} t\left(z_{n_{1}}^{2 \sigma}+z_{n_{1}}^{2 \sigma}\right)}\left(c_{n_{1}} d_{n_{2}}\right)\left(e_{n_{1}} e_{n_{2}}\right)\right|^{2} d x d t
\end{aligned}
$$

where

$$
\# \Lambda_{N_{1}, N_{2}, \tau}=\#\left\{\left(n_{1}, n_{2}\right) \in \mathbb{N}^{2}: n_{1} \sim N_{1}, n_{2} \sim N_{2},\left|z_{n_{1}}^{2 \sigma}+z_{n_{2}}^{2 \sigma}-\tau\right| \leq \frac{1}{2}\right\} .
$$

By expanding the square above and using Plancherel, we have

$$
\begin{aligned}
& \text { (5.6) }=\int_{\mathbb{R} \times B^{d}} \eta(t) \sum_{\tau, \tau^{\prime}} \sum_{\substack{\left(n_{1}, n_{2}\right) \in \Lambda_{N_{1}, N_{2}, \tau} \\
\left(n_{1}^{\prime}, n_{2}^{\prime}\right) \in \Lambda_{N_{1}^{\prime}, N_{2}^{\prime}, \tau^{\prime}}}} e^{\mathrm{it}\left(z_{n_{1}^{\prime}}^{2 \sigma}+z_{n_{2}^{\prime}}^{2 \sigma}-z_{n_{1}}^{2 \sigma}-z_{n_{2}}^{2 \sigma}\right)}\left(c_{n_{1}} d_{n_{2}}\right)\left(\overline{c_{n_{1}^{\prime}} d_{n_{2}^{\prime}}}\right)\left(e_{n_{1}} e_{n_{2}}\right)\left(e_{n_{1}^{\prime}} e_{n_{2}^{\prime}}\right) d x d t \\
& =\sum_{\tau, \tau^{\prime}} \sum_{\substack{\left(n_{1}, n_{2}\right) \in \Lambda_{N_{1}, N_{2}, \tau} \\
\left(n_{1}^{\prime}, n_{2}^{\prime}\right) \in \Lambda_{N_{1}^{\prime}, N_{2}^{\prime}, \tau^{\prime}}}} \widehat{\eta}\left(\left(z_{n_{1}^{\prime}}^{2 \sigma}+z_{n_{1}^{\prime}}^{2 \sigma}\right)-\left(z_{n_{1}}^{2 \sigma}+z_{n_{2}}^{2 \sigma}\right)\right)\left(c_{n_{1}} d_{n_{2}}\right)\left(\overline{c_{n_{1}^{\prime}} d_{n_{2}^{\prime}}}\right) \int_{B^{d}}\left(e_{n_{1}} e_{n_{2}}\right)\left(e_{n_{1}^{\prime}} e_{n_{2}^{\prime}}\right) d x \\
& \lesssim \sum_{\tau, \tau^{\prime}} \frac{1}{1+\left|\tau-\tau^{\prime}\right|^{2}} \sum_{\substack{n, \sim N_{1}, n_{2} \sim N_{2} \\
n \\
n_{1}^{\prime} \sim N_{1}^{\prime}, n_{2}^{\prime} \sim N_{2}^{\prime}}} \mathbf{1}_{\Lambda_{N_{1}, N_{2}, \tau}} \mathbf{1}_{\Lambda_{N_{1}^{\prime}, N_{2}^{\prime}, \tau^{\prime}}}\left(c_{n_{1}} d_{n_{2}}\right)\left(\overline{c_{n_{1}^{\prime}} d_{n_{2}^{\prime}}}\right)\left\|e_{n_{1}} e_{n_{2}}\right\|_{L^{2}\left(B^{d}\right)}\left\|e_{n_{1}^{\prime}} e_{n_{2}^{\prime}}\right\|_{L^{2}\left(B^{d}\right)}
\end{aligned}
$$

Then by Schur's test, we arrive at

$$
\begin{aligned}
(5.7) & \lesssim \sum_{\tau \in \mathbb{N}}\left(\sum_{\left(n_{1}, n_{2}\right) \in \Lambda_{N_{1}, N_{2}, \tau}}\left|c_{n_{1}} d_{n_{2}}\right|\left\|e_{n_{1}} e_{n_{2}}\right\|_{L^{2}\left(B^{d}\right)}\right)^{2} \\
& \lesssim \sum_{\tau \in \mathbb{N}} \# \Lambda_{N_{1}, N_{2}, \tau} \sum_{\left(n_{1}, n_{2}\right) \in \Lambda_{N_{1}, N_{2}, \tau}}\left|c_{n_{1}} d_{n_{2}}\right|^{2}\left\|e_{n_{1}} e_{n_{2}}\right\|_{L^{2}\left(B^{d}\right)}^{2}
\end{aligned}
$$

We claim that

Claim 5.7 (1) \# $\Lambda_{N_{1}, N_{2}, \tau}=\mathscr{O}\left(N_{2}\right)$; 
(2) $\left\|e_{n_{1}} e_{n_{2}}\right\|_{L^{2}\left(B^{d}\right)}^{2} \lesssim N_{2}^{d-2+}$.

Assuming Claim 5.7, we see that

$$
(5.8) \lesssim \sum_{\tau \in \mathbb{N}} N_{2}^{d-1+} \sum_{\left(n_{1}, n_{2}\right) \in \Lambda_{N_{1}, N_{2}, \tau}}\left|c_{n_{1}} d_{n_{2}}\right|^{2} \lesssim N_{2}^{d-1+\varepsilon}\left\|u_{1}\right\|_{L^{2}\left(B^{d}\right)}^{2}\left\|u_{2}\right\|_{L^{2}\left(B^{d}\right)}^{2}
$$

Therefore, (5.1) follows.

Now we are left to prove Claim 5.7.

Proof of Claim 5.7 In fact, (2) is due to Hölder inequality and the logarithmic bound on the $L^{p}$ norm of $e_{n}$ in (2.1)

$$
\left\|e_{n_{1}} e_{n_{2}}\right\|_{L^{2}\left(B^{d}\right)} \lesssim\left\|e_{n_{1}}\right\|_{L^{\frac{2 d}{d-1}-\left(B^{d}\right)}}\left\|e_{n_{2}}\right\|_{L^{2 d+}\left(B^{d}\right)} \lesssim n_{2}^{\frac{d-1}{2}-\frac{d}{2 d}+}=n_{2}^{\frac{d-2}{2}+} .
$$

For (1), we have that for fixed $\tau \in \mathbb{N}$ and fixed $n_{2} \sim N_{2}$

$$
\left|z_{n_{1}}^{2 \sigma}+z_{n_{2}}^{2 \sigma}-\tau\right| \leq \frac{1}{2} \Longrightarrow z_{n_{1}} \in\left[\left(\tau-\frac{1}{2}-z_{n_{2}}^{2 \sigma}\right)^{\frac{1}{2 \sigma}},\left(\tau+\frac{1}{2}-z_{n_{2}}^{2 \sigma}\right)^{\frac{1}{2 \sigma}}\right]
$$

There are at most 1 integer $z_{n_{1}}$ in this interval, since by convexity

$$
\left(\tau+\frac{1}{2}-z_{n_{2}}^{2 \sigma}\right)^{\frac{1}{2 \sigma}}-\left(\tau-\frac{1}{2}-z_{n_{2}}^{2 \sigma}\right)^{\frac{1}{2 \sigma}} \leq 1^{\frac{1}{2 \sigma}}=1
$$

Then

$\# \Lambda_{N_{1}, N_{2}, \tau}=\#\left\{\left(n_{1}, n_{2}\right) \in \mathbb{N}^{2}: n_{1} \sim N_{1}, n_{2} \sim N_{2},\left|z_{n_{1}}^{2 \sigma}+z_{n_{2}}^{2 \sigma}-\tau\right| \leq \frac{1}{2}\right\} \sim \mathscr{O}\left(N_{2}\right)$

We finish the proof of Claim 5.7.

The estimation of (5.2) is similar, hence omitted.

The proof of Lemma 5.3 is complete now.

\subsection{Nonlinear estimates}

In the local theory, we need a nonlinear estimate of the following form

$$
\int_{\mathbb{R}} \int_{\Theta} u_{0} u_{1} u_{2} u_{3} d x d t \leq c\left\|u_{0}\right\|_{X_{\sigma}^{-s, b^{\prime}}\left(\mathbb{R} \times B^{d}\right)} \prod_{j=1}^{3}\left\|u_{j}\right\|_{X_{\sigma}^{s, b^{\prime}}\left(\mathbb{R} \times B^{d}\right)},
$$

where $u_{j} \in\{u, \bar{u}\}$ for $j=1,2,3$.

To this end, we first study the nonlinear behavior of all frequency localized $u_{j}$ 's based on the bilinear estimates that we obtained, then sum over all frequencies. 
For $j \in\{0,1,2,3\}$, let $N_{j}=2^{k}, k \in \mathbb{N}$. We denote

$$
u_{j}=\sum_{N_{j} \leq\left\langle z_{n}\right\rangle<2 N_{j}} P_{z_{n}} u_{j}
$$

Also we denote by $\underline{N}=\left(N_{0}, N_{1}, N_{2}, N_{3}\right)$ the quadruple of $2^{k}$ numbers, $k \in \mathbb{N}$, and

$$
L(\underline{N})=\int_{\mathbb{R} \times B^{d}} \prod_{j=0}^{3} u_{j} d x d t
$$

Lemma 5.8 (Localized nonlinear estimates) Assume that $N_{1} \geq N_{2} \geq N_{3}$. Then there exists $0<b^{\prime}<\frac{1}{2}$ such that one has

$$
\begin{gathered}
|L(\underline{N})| \lesssim\left(N_{2} N_{3}\right)^{\frac{d-1}{2}+\varepsilon} \prod_{j=0}^{3}\left\|u_{j}\right\|_{X_{\sigma}^{0, b^{\prime}}}, \\
|L(\underline{N})| \lesssim\left(\frac{N_{1}}{N_{0}}\right)^{2}\left(N_{2} N_{3}\right)^{\frac{d-1}{2}+\varepsilon} \prod_{j=0}^{3}\left\|u_{j}\right\|_{X_{\sigma}^{0, b^{\prime}}}
\end{gathered}
$$

Remark 5.9 Lemma 5.8 will play an important role in the local theory. Moreover, the first estimate (5.9) will be used in the case $N_{0} \leq c N_{1}$ while the second one will be used in the case $N_{0} \geq c N_{1}$. The proof of Lemma 5.8 is adapted from Lemma 2.5 in [1]. We briefly present the proof, since a treatment in this proof will be used in the next section.

Proof of Lemma 5.8 We start with (5.9).

On one hand, by Hölder inequality, Bernstein inequality and Lemma 2.2, we write

$$
|L(\underline{N})| \lesssim\left\|u_{0}\right\|_{L_{t}^{4} L_{x}^{2}}\left\|u_{1}\right\|_{L_{t}^{4} L_{x}^{2}}\left\|u_{2}\right\|_{L_{t}^{4} L_{x}^{\infty}}\left\|u_{3}\right\|_{L_{t}^{4} L_{x}^{\infty}} \lesssim\left(N_{2} N_{3}\right)^{\frac{d}{2}} \prod_{j=0}^{3}\left\|u_{j}\right\|_{X_{\sigma}^{0, \frac{1}{4}}} .
$$

On the other hand, we can estimate $|L(\underline{N})|$ using Proposition 5.6. That is,

$$
|L(\underline{N})| \lesssim\left\|u_{0} u_{2}\right\|_{L_{t, x}^{2}}\left\|u_{1} u_{3}\right\|_{L_{t, x}^{2}} \lesssim\left(N_{2} N_{3}\right)^{\frac{d-1}{2}+\varepsilon} \prod_{j=0}^{3}\left\|u_{j}\right\|_{X_{\sigma}^{0, b_{0}}}
$$

where $b_{0}>\frac{1}{2}$.

Interpolation between the two estimates above implies

$$
|L(\underline{N})| \lesssim\left(N_{2} N_{3}\right)^{\frac{d-1}{2}+\varepsilon^{\prime}} \prod_{j=0}^{3}\left\|u_{j}\right\|_{X_{\sigma}^{0, b^{\prime}}}
$$


where $b^{\prime} \in\left(0, \frac{1}{2}\right)$ and $\varepsilon^{\prime}$ is a small positive power after interpolation. This finishes the computation of (5.9).

For (5.10), we will use a trick to introduce a Laplacian operator into the integral. This is the treatment that we mentioned earlier that will be used in the next section.

First recall Green's theorem,

$$
\int_{B^{d}} \Delta f g-f \Delta g d x=\int_{\mathbb{S}^{d-1}} \frac{\partial f}{\partial v} g-f \frac{\partial g}{\partial v} d \sigma
$$

Note that

$$
-\Delta e_{k}=z_{k}^{2} e_{k}
$$

where $z_{k}^{2}$,s are the eigenvalues defined in (2.2). Then we write

$$
u_{0}=-\frac{\Delta}{N_{0}^{2}} \sum_{z_{n_{0}} \sim N_{0}} c_{n_{0}}\left(\frac{N_{0}}{z_{n_{0}}}\right)^{2} e_{n_{0}}
$$

Define

$$
T u_{0}=\sum_{z_{n_{0}} \sim N_{0}} c_{n_{0}}\left(\frac{N_{0}}{z_{n_{0}}}\right)^{2} e_{n_{0}}, \quad V u_{0}=\sum_{z_{n_{0}} \sim N_{0}} c_{n_{0}}\left(\frac{z_{n_{0}}}{N_{0}}\right)^{2} e_{n_{0}} .
$$

It is easy to see that for all $s$

$$
\begin{aligned}
T V u_{0} & =V T u_{0}=u_{0}, \\
\left\|T u_{0}\right\|_{H_{x}^{s}} & \sim\left\|u_{0}\right\|_{H_{x}^{s}} \sim\left\|V u_{0}\right\|_{H_{x}^{s}} .
\end{aligned}
$$

Using this notation, we write

$$
u_{0}=-\frac{\Delta}{N_{0}^{2}} T u_{0}
$$

and

$$
L(\underline{N})=\frac{1}{N_{0}^{2}} \int_{\mathbb{R} \times B^{d}} T u_{0} \Delta\left(\prod_{j=1}^{3} u_{j}\right)
$$

By the product rule and the assumption that $N_{1} \geq N_{2} \geq N_{3}$, we only need to consider the two largest cases of $\Delta\left(u_{1} u_{2} u_{3}\right)$. They are

(1) $\left(\Delta u_{1}\right) u_{2} u_{3}$

(2) $\left(\nabla u_{1}\right) \cdot\left(\nabla u_{2}\right) u_{3}$. 
We denote

$$
\begin{aligned}
& J_{11}(\underline{N})=\int_{\mathbb{R} \times B^{d}} T u_{0}\left(\Delta u_{1}\right) u_{2} u_{3}, \\
& J_{12}(\underline{N})=\int_{\mathbb{R} \times B^{d}} T u_{0}\left(\nabla u_{1}\right) \cdot\left(\nabla u_{2}\right) u_{3} .
\end{aligned}
$$

Using $\Delta u_{i}=-N_{i}^{2} V u_{i}$, we obtain

$$
\frac{1}{N_{0}^{2}}\left|J_{11}(\underline{N})\right| \lesssim\left(\frac{N_{1}}{N_{0}}\right)^{2}\left(N_{2} N_{3}\right)^{\frac{d-1}{2}+\varepsilon} \prod_{j=0}^{3}\left\|u_{j}\right\|_{X_{\sigma}^{0, b^{\prime}}}
$$

Now for $\left|J_{12}(\underline{N})\right|$, we estimate it in a similar fashion that we did in (5.9). On one hand, by Hölder inequality, Bernstein inequality and Lemma 2.2, we have

$$
\left|J_{12}(\underline{N})\right| \lesssim N_{1} N_{2}\left(N_{2} N_{3}\right)^{\frac{d}{2}} \prod_{j=0}^{3}\left\|u_{j}\right\|_{X_{\sigma}^{0, \frac{1}{4}}} \cdot
$$

On the other hand, using Proposition 5.6, we get

$$
\left|J_{12}(\underline{N})\right| \lesssim\left\|\nabla u_{1} u_{3}\right\|_{L_{t, x}^{2}}\left\|\nabla u_{2} T u_{0}\right\|_{L_{t, x}^{2}} \lesssim N_{1} N_{2}\left(N_{2} N_{3}\right)^{\frac{d-1}{2}+\varepsilon} \prod_{j=0}^{3}\left\|u_{j}\right\|_{X_{\sigma}^{0, b_{0}}} .
$$

Interpolation between the two estimates above implies

$$
\frac{1}{N_{0}^{2}}\left|J_{12}(\underline{N})\right| \lesssim \frac{N_{1} N_{2}}{N_{0}^{2}}\left(N_{2} N_{3}\right)^{\frac{d-1}{2}+\varepsilon^{\prime}} \prod_{j=0}^{3}\left\|u_{j}\right\|_{X_{\sigma}^{0, b^{\prime}}}
$$

Similarly $\varepsilon^{\prime}$ is a small positive power after interpolation. The proof of Lemma 5.8 is complete.

Proposition 5.10 (Nonlinear estimates) For $\sigma \in\left[\frac{1}{2}, 1\right], s>s_{l}(\sigma)$ as in (1.3), there exist $b, b^{\prime} \in \mathbb{R}$ satisfying

$$
0<b^{\prime}<\frac{1}{2}<b, \quad b+b^{\prime}<1
$$

such that for every triple $\left(u_{1}, u_{2}, u_{3}\right)$ in $X_{\sigma}^{s, b}\left(\mathbb{R} \times B^{d}\right)$,

$$
\left\||u|^{2} u\right\|_{X_{\sigma}^{s,-b^{\prime}}\left(\mathbb{R} \times B^{d}\right)} \lesssim \prod_{j=1}^{3}\|u\|_{X_{\sigma}^{s, b}\left(\mathbb{R} \times B^{d}\right)}^{3}
$$


Proof of Proposition 5.10 Based on Lemma 5.8, we only need to consider $L=$ $\sum_{N} L(\underline{N})$. By symmetry, we can reduce the sum into the following two cases:

(1) $N_{0} \leq c N_{1}$

(2) $N_{0} \geq c N_{1}$.

Case 1: $N_{0} \leq c N_{1}$.

Using Lemma 5.8 and Cauchy-Schwarz inequality, we obtain

$$
\begin{aligned}
\mid \sum L(\underline{N)} \mid & \lesssim \sum_{\substack{N_{1} \geq N_{2} \geq N_{3} \\
N_{0} \leq c N_{1}}}\left(N_{2} N_{3}\right)^{\frac{d-1}{2}+\varepsilon}\left\|v_{0}\right\|_{X_{\sigma}^{0, b^{\prime}}} \prod_{j=1}^{3}\left\|u_{j}\right\|_{X_{\sigma}^{0, b^{\prime}}} \\
& \lesssim \sum_{\substack{N_{1} \geq N_{2} \geq N_{3} \\
N_{0} \leq c N_{1}}} \frac{N_{0}^{s}}{N_{1}^{s}}\left(N_{2} N_{3}\right)^{-s+\frac{d-1}{2}+\varepsilon}\left\|v_{0}\right\|_{X_{\sigma}^{-s, b^{\prime}}} \prod_{j=1}^{2 q+1}\left\|u_{j}\right\|_{X_{\sigma}^{s, b^{\prime}}} \\
& \lesssim\|v\|_{X_{\sigma}^{-s, b^{\prime}}}\|u\|_{X_{\sigma}^{s, b^{\prime}}}^{3},
\end{aligned}
$$

where $s>s_{l}(\sigma)$. For the last inequality, we sum from the smallest index $N_{3}$ to the largest one using Cauchy-Schwarz inequality. Then by the embedding $X_{\sigma}^{s, b} \subset X_{\sigma}^{s, b^{\prime}}$ and duality, we have the estimate in Case 1.

Case 2: $N_{0} \geq c N_{1}$.

Similarly, by Lemma 5.8 and Cauchy-Schwarz inequality again, we have

$$
\begin{aligned}
\mid \sum L(\underline{N)} \mid & \lesssim \sum_{\substack{N_{1} \geq N_{2} \geq N_{3} \\
N_{0} \geq c N_{1}}}\left(\frac{N_{1}}{N_{0}}\right)^{2}\left(N_{2} N_{3}\right)^{\frac{d-1}{2}+\varepsilon}\left\|v_{0}\right\|_{X_{\sigma}^{0, b^{\prime}}} \prod_{j=1}^{3}\left\|u_{j}\right\|_{X_{\sigma}^{0, b^{\prime}}} \\
& \lesssim \sum_{\substack{N_{1} \geq N_{2} \geq N_{3} \\
N_{0} \geq c N_{1}}}\left(\frac{N_{1}}{N_{0}}\right)^{2-s} \frac{N_{0}^{s}}{N_{1}^{s}}\left(N_{2} N_{3}\right)^{-s+\frac{d-1}{2}+\varepsilon}\left\|v_{0}\right\|_{X_{\sigma}^{-s, b^{\prime}}} \prod_{j=1}^{3}\left\|u_{j}\right\|_{X_{\sigma}^{s, b^{\prime}}} \\
& \lesssim\left\|v_{0}\right\|_{X_{\sigma}^{-s, b^{\prime}}}\|u\|_{X_{\sigma}^{s, b^{\prime}}}^{3}
\end{aligned}
$$

where $s>s_{l}(\sigma)$. Then by the embedding $X_{\sigma}^{s, b} \subset X_{\sigma}^{s, b^{\prime}}$ and duality, we have the estimate in Case 2. The proof of Proposition 5.10 is complete.

\subsection{Local well-posedness}

We now turn to the proof of the main result in this section: local well-posedness.

Proof of Theorem 5.1 Let $u_{0} \in H^{s}$. We first define a map

$$
F(u)(t):=S_{\sigma}(t) u_{0}-\mathbf{i} \int_{0}^{t} S_{\sigma}(t-s)|u|^{2} u d s .
$$


We prove this locally well-posedness theory using a standard fixed point argument. Let $R_{0}>0$ and $u_{0} \in H^{s}\left(B^{d}\right)$ with $\left\|u_{0}\right\|_{H^{s}} \leq R_{0}$. We show that there exists $R>0$ and $0<\delta=\delta\left(R_{0}\right)<1$ such that $F$ is a contraction mapping from $B(0, R) \subset X_{\sigma, \delta}^{s, b}\left(B^{d}\right)$ onto itself.

Define $R=2 c_{0} R_{0}$.

(1) $F$ is a self map from $B(0, R) \subset X_{\sigma, \delta}^{s, b}\left(B^{d}\right)$ onto itself.

For $\delta<1$, by Lemma 2.4, Proposition 5.10 and Sobolev embedding

$$
\begin{aligned}
\|F(u)\|_{X_{\sigma, \delta}^{s, b}\left(B^{d}\right)} & \leq c_{0}\left\|u_{0}\right\|_{H^{s}}+c_{1} \delta^{1-b-b^{\prime}}\left\||u|^{2} u\right\|_{X_{\sigma, \delta}^{s,-b^{\prime}}\left(B^{d}\right)} \\
& \leq c_{0}\left\|u_{0}\right\|_{H^{s}}+c_{2} \delta^{1-b-b^{\prime}} \delta^{3\left(b-b^{\prime}\right)}\|u\|_{X_{\sigma, \delta}^{s, b}\left(B^{d}\right)}^{3} \\
& \leq c_{0}\left\|u_{0}\right\|_{H^{s}}+c_{2} \delta^{1+2 b-4 b^{\prime}} R^{3} .
\end{aligned}
$$

Taking $\delta_{1}=\left(\frac{c_{0}}{c_{2} R^{2}}\right)^{\frac{1}{1+2 b-4 b^{\prime}}}<1$ such that $c_{2} \delta_{1}^{1+2 b-4 b^{\prime}} R^{3}=c_{0} R$, we see that $F$ is a self map.

(2) $F$ is a contraction mapping.

Similarly, by Lemma 2.4 and Proposition 5.10

$$
\begin{aligned}
\|F(u)-F(v)\|_{X_{\sigma, \delta}^{s, b}\left(B^{d}\right)} & \leq c_{3} \delta^{1+2 b-4 b^{\prime}}\left(\|u\|_{X_{\sigma, \delta}^{s, b}\left(B^{d}\right)}^{2}+\|v\|_{X_{\sigma, \delta}^{s, b}\left(B^{d}\right)}^{2}\right)\|u-v\|_{X_{\sigma, \delta}^{s, b}\left(B^{d}\right)} \\
& \leq c_{4} \delta^{1+2 b-4 b^{\prime}} R^{2}\|u-v\|_{X_{\sigma, \delta}^{s, b}\left(B^{d}\right)} .
\end{aligned}
$$

Taking $\delta_{2}=\left(\frac{1}{2 c_{4} R^{2}}\right)^{\frac{1}{1+2 b-4 b^{\prime}}}$ such that $c_{4} \delta_{2}^{1+2 b-4 b^{\prime}} R^{2}=\frac{1}{2}$, we can make $F$ a contraction mapping.

Now we choose

$$
\delta_{R}=\min \left\{\delta_{1}, \delta_{2}\right\}
$$

As a consequence of the fixed point argument, we have

$$
\|u\|_{X_{\sigma, \delta}^{s, b}\left(B^{d}\right)} \leq 2 c_{0}\left\|u_{0}\right\|_{H^{s}}
$$

(3) Stability.

If $u$ and $v$ are two solutions to (1.1) with initial data $u(0)=u_{0}$ and $v(0)=v_{0}$ respectively. Then by (5.11) and the choice of $\delta_{R}(5.12)$

$$
\|u-v\|_{X_{\sigma, \delta}^{s, b}\left(B^{d}\right)} \leq c_{0}\left\|u_{0}-v_{0}\right\|_{H^{s}}+c_{4} \delta^{1+2 b-4 b^{\prime}} R^{2}\|u-v\|_{X_{\sigma, \delta}^{s, b}\left(B^{d}\right)},
$$

which implies

$$
\|u-v\|_{X_{\sigma, \delta}^{s, b}\left(B^{d}\right)} \leq c\left\|u_{0}-v_{0}\right\|_{H^{s}}
$$


Now the proof of Theorem 5.1 is finished.

Remark 5.11 For finite $N$ let us denote $\Pi^{N} L^{2}$ by $E_{N}$ and the local flow on $E_{N}=$ $\Pi^{N} L^{2}$ by $\phi_{t}^{N}$. We verify easily that the local existence time obtained in Theorem 5.1 is valid for the Galerkin approximations for the same radius $R$ of the balls. We set $\phi_{t}$ to be the local flow constructed in Theorem 5.1 in $X_{\sigma}^{s, b}$.

Proposition 5.12 (Convergence of Galerkin projections to FNLS) Let $s>s_{l}(\sigma)$ as in (1.3), $u_{0} \in H^{s}$ and $\left(u_{0}^{N}\right)$ be a sequence that converges to $u_{0}$ in $H^{s}$, where $u_{0}^{N} \in E_{N}$. Then for any $r \in\left(s_{l}(\sigma), s\right)$

$$
\left\|\phi_{t}^{N} u_{0}^{N}-\phi_{t} u_{0}\right\|_{X_{\sigma, T}^{r, b}} \rightarrow 0 \quad \text { as } N \rightarrow \infty .
$$

Proof of Proposition 5.12 Since $u_{0}^{N} \rightarrow u_{0}$, there exists an $R$ such that $u_{0}^{N} \in B_{R}\left(H^{\gamma}\right)$. Take the same $T_{R}$ as in (5.12). Set $w_{0}^{N}=u_{0}^{N}-u_{0}$ and $w^{N}=\phi_{t}^{N} u_{0}^{N}-\phi_{t} u_{0}$, then we write

$$
\begin{aligned}
w^{N}= & S_{\sigma}(t) w_{0}^{N}-\mathbf{i} \int_{0}^{t} S_{\sigma}(t-s)\left(\Pi^{N}\left|\phi_{t}^{N} u_{0}^{N}\right|^{2} \phi_{t}^{N} u_{0}^{N}-\left|\phi_{t} u_{0}\right|^{2} \phi_{t} u_{0}\right) d s \\
= & S_{\sigma}(t) w_{0}^{N}-\mathbf{i} \int_{0}^{t} S_{\sigma}(t-s) \Pi^{N}\left(\left|\phi_{t}^{N} u_{0}^{N}\right|^{2} \phi_{t}^{N} u_{0}^{N}-\left|\phi_{t} u_{0}\right|^{2} \phi_{t} u_{0}\right) \\
& -\left(1-\Pi^{N}\right)\left(\left|\phi_{t} u_{0}\right|^{2} \phi_{t} u_{0}\right) d s .
\end{aligned}
$$

Using Hölder inequality, Lemma 2.4, Proposition 5.10 and the choice of $T_{R}$ as in (5.12), we have

$$
\begin{aligned}
\left\|w^{N}\right\|_{X_{\sigma, T}^{r, b}} & \leq\left\|w_{0}^{N}\right\|_{H^{s}}+C\left\|w^{N}\right\|_{X_{\sigma, T}^{r, b}}\left(\left\|u^{N}\right\|_{X_{\sigma, T}^{r, b}}^{2}+\|u\|_{X_{\sigma, T}^{r, b}}^{2}\right)+\left\|\left(1-\Pi^{N}\right)|u|^{2} u\right\|_{X_{\sigma, T}^{r, b}} \\
& \leq\left\|w_{0}^{N}\right\|_{H^{s}}+C\left\|w^{N}\right\|_{X_{\sigma, T}^{r, b}}\left(\left\|u^{N}\right\|_{X_{\sigma, T}^{r, b}}^{2}+\|u\|_{X_{\sigma, T}^{r, b}}^{2}\right)+C_{1} z_{N}^{\frac{r-s}{2}}\|u\|_{X_{\sigma, T}^{s, b}}^{3} .
\end{aligned}
$$

Therefore,

$$
\begin{aligned}
\left\|w^{N}\right\|_{X_{\sigma, T}^{r, b}} & \leq\left\|w_{0}^{N}\right\|_{H^{s}}+\frac{1}{2}\left\|w^{N}\right\|_{X_{\sigma, T}^{r, b}}+C_{2} z_{N}^{\frac{\sigma-s}{2}} \\
\left\|w^{N}\right\|_{X_{\sigma, T}^{r, b}} & \leq 2\left\|w_{0}^{N}\right\|_{H^{s}}+2 C_{2} z_{N}^{\frac{r-s}{2}}
\end{aligned}
$$

Recall that $z_{N}^{2} \sim N^{2}$, then

$$
\left\|w^{N}\right\|_{X_{\sigma, T}^{r, b}} \rightarrow 0 \quad \text { as } N \rightarrow \infty
$$

Hence the proof of Proposition 5.12 is finished.

Remark 5.13 In Sect. 4, we fulfilled Assumptions 4 to 7 for any $\sigma \in(0,1]$. However, Theorem 5.1 covers only the range $\sigma \in\left[\frac{1}{2}, 1\right]$ and the given regularities. The case 
$\sigma \in\left(0, \frac{1}{2}\right)$ was not treated in Theorem 5.1 because of the convexity needed in the proof of Claim 5.7. Nevertheless, in smooth regularities $s>\frac{d}{2}$ a same local well-posedness as in Theorem 5.1 for $\sigma \in\left(0, \frac{1}{2}\right)$ holds true. We will not present the details of such easy argument. We also readily have the convergence established in Proposition (5.12) for such values of $\sigma$. This ends the proof of Theorem 1.1.

\section{Proof of Theorem 1.3}

In this section we present the proof of Theorem 1.3. We consider then $\sigma \in(0,1]$ and $\max \left(\sigma, \frac{1}{2}\right) \leq s \leq 1+\sigma$, or $\sigma \in\left(\frac{1}{2}, 1\right]$ and $s \in(0, \sigma]$, with $d \geq 2$. Recall that from (4.7)

$$
\int_{L^{2}}\|u\|_{H^{s}}^{2}+\left\||u|^{2}\right\|_{\dot{H}^{\sigma}}^{2}+\left\||u|^{2} u\right\|_{L^{2}}^{2} \mu^{N}(d u) \leq C,
$$

where $C$ does not depend on $N$.

\subsection{Existence of global solutions and invariance of the measure}

For an arbitrary positive integer $k$, let us consider the spaces

$$
\begin{aligned}
X_{k} & =L^{2}\left([0, k], H^{s}\right) \cap\left(H^{1}\left([0, k], H^{s-2 \sigma}\right)+H^{1}\left([0, k], L^{2}\right)\right)=: X_{k}^{1}+X_{k}^{2} ; \\
Y_{k} & =L^{2}\left([0, k], H^{s-}\right) \cap C\left([0, k], H^{(s-2 \sigma)-}\right) .
\end{aligned}
$$

Combining standard embedding results we obtain the compact embedding $X_{k} \subset \subset Y_{k}$.

Denote by $v_{k}^{N}$ the laws of the processes $\left(u^{N}(t)\right)_{t \in[0, k)}$ that are seen as random variables valued in $C\left([0, k], H^{(s-2 \sigma)-}\right)$. We see, using the invariance, the relation between $\mu^{N}$ and $v_{k}^{N}$ :

$$
\mu^{N}=\left.v_{k}^{N}\right|_{t=t_{0}} \quad \text { for any } t_{0} \in[0, k]
$$

Proposition 6.1 We have the estimate

$$
\int_{X_{k}}\|u\|_{X_{k}}^{2} v_{k}^{N}(d u) \leq C,
$$

where $C$ does not depend on $N$.

Proof of Proposition 6.1 Let us write the fractional NLS (4.1) in the integral form

$$
u^{N}(t)=u^{N}(0)-\mathbf{i} \int_{0}^{t}(-\Delta)^{\sigma} u^{N} d s-\mathbf{i} \int_{0}^{t} \Pi^{N}\left|u^{N}\right|^{2} u^{N} d s .
$$


Now using (6.1) we have that

$$
\begin{aligned}
& \mathbb{E}\left\|\int_{0}^{t}(-\Delta)^{\sigma} u^{N} d s\right\|_{H^{1}\left([0, k], H^{s-2 \sigma}\right)}^{2} \leq \mathbb{E} \int_{0}^{k}\left\|u^{N}\right\|_{H^{s}}^{2} d s \leq C k \\
& \mathbb{E}\left\|\int_{0}^{t} \Pi^{N}\left|u^{N}\right|^{2} u^{N} d s\right\|_{H^{1}\left([0, k], L^{2}\right)}^{2} \leq \mathbb{E} \int_{0}^{k}\left\|\Pi^{N}\left|u^{N}\right|^{2} u^{N}\right\|_{L^{2}}^{2} d s \leq C k .
\end{aligned}
$$

We recall that,

$$
\mathbb{E} \int_{0}^{k}\left\|u^{N}\right\|_{H^{s}}^{2} d s \leq C k .
$$

Combining the above estimates we obtain Proposition 6.1.

Proposition $6.2 u^{N}$ is compact in $Y_{k} \mathbb{P}$-almost surely.

Proof of Proposition 6.2 Combining Proposition (6.1) and the Prokhorov theorem (Theorem A.4) we obtain a weak compactness of $\left(v_{k}^{N}\right)$. Thanks to the Skorokhod representation theorem (see Theorem A.5), there are random variables $\tilde{u}_{k}^{N}$ and $\tilde{u}_{k}$, defined on a same probability space that is denoted by $(\Omega, \mathbb{P})$, such that

(1) $\tilde{u}_{k}^{N} \rightarrow \tilde{u_{k}}$ weakly on $X_{k}$,

(2) $\tilde{u}_{k}^{N}$ and $\tilde{u}_{k}$ are distributed by $v_{k}^{N}$ and $v_{k}$ respectively.

The proof of Proposition 6.2 is finished.

Proposition $6.3 \Pi^{N}\left|u^{N}\right|^{2} u^{N}$ converges to $|u|^{2} u$ in $L_{t}^{1} H^{-\frac{d}{2}-1}$ as $N \rightarrow \infty$.

(1) $u^{N}$ converges to $u$ almost surely in $L_{t}^{2} H^{s-} \subset L_{t, x}^{2}$

(2) $\left|u^{N}\right|^{2}$ converges almost surely in $L_{t}^{2} H^{\sigma-} \subset L_{t, x}^{2}$. In particular $\left|u^{N}\right|^{2}$ is bounded in $L_{t, x}^{2}$ almost surely, i.e $\left\|u^{N}\right\|_{L_{t, x}^{4}}$ is bounded $\mathbb{P}$-almost surely.

Now let us write, by using $\Pi^{N}\left|u^{N}\right|^{2} u^{N}=\left|u^{N}\right|^{2} u^{N}-\Pi^{>N}\left|u^{N}\right|^{2} u^{N}$, a Bernstein inequality and convergence and boundedness established above,

$$
\begin{aligned}
\left\|\Pi^{N}\left|u^{N}\right|^{2} u^{N}-|u|^{2} u\right\|_{L_{t}^{1} H^{-\frac{d}{2}-1}} \leq & C\left\|\left|u^{N}\right|^{2} u^{N}-|u|^{2} u\right\|_{L_{t, x}^{1}} \\
& +\left\|\Pi^{>N}\left|u^{N}\right|^{2} u^{N}\right\|_{L_{t}^{1} H^{-\frac{d}{2}-1}} \\
\leq & \left\|u^{N}-u\right\|_{L_{t, x}^{2}}\left(\left\|\left|u^{N}\right|^{2}\right\|_{L_{t, x}^{2}}+\left\||u|^{2}\right\|_{L_{t, x}^{2}}\right) \\
& +N^{-1}\left\|\left|u^{N}\right|^{2} u^{N}\right\|_{L_{t}^{1} H^{-\frac{d}{2}}} \\
\leq & \tilde{C}(\omega)\left\|u^{N}-u\right\|_{L_{t, x}^{2}}+C(k) N^{-1}\left\|\left|u^{N}\right|^{2} u^{N}\right\|_{L_{t}^{\frac{4}{3}}} \frac{4}{L_{x}^{3}} \\
& \lesssim \omega, k\left\|u^{N}-u\right\|_{L_{t, x}^{2}}+N^{-1}\left\|u^{N}\right\|_{L_{t, x}^{4}}^{3} \\
& \lesssim \omega, k\left\|u^{N}-u\right\|_{L_{t, x}^{2}}+N^{-1} .
\end{aligned}
$$


Combining Propositions 6.2 and 6.3 with a diagonal argument we obtain the almost sure existence of global solutions for FNLS with $\sigma \in(0,1), 0<s \leq 1+\sigma$ and $d \geq 2$.

For the invariance of the law of the constructed solution $u(t)$, let us denote by $v$ the law of the process $u=(u(t))_{t \in \mathbb{R}}$. From the subsequence in the $N$-parameter that produced the limiting measure $v$, we can extract a subsequence, using the Prokhorov theorem and (6.1), that produces a measure $\mu$ as a weak limit point of $\left(\mu^{N}=\left.v^{N}\right|_{t=t_{0}}\right)$. Passing to the limit along this subsequence in the relation (6.2), we see that $\mu=\left.v\right|_{t=t_{0}}$ for any $t_{0} \in \mathbb{R}$. This implies that $\mu$ is an invariant law for $u$.

\subsection{Uniqueness and Continuity}

The uniqueness argument is formulated in the following observation.

Proposition 6.4 The space $\Lambda=C_{t} L_{x}^{2} \cap L_{t}^{2} H^{\frac{1+}{2}}\left(B^{d}\right)$ is a uniqueness class for the fractional NLS (1.1).

Proof of Proposition 6.4 Let $u, v \in \Lambda$ be two solutions to (1.1), both starting at $u_{0}$. Let us write the Duhamel formulation

$$
u(t)=S_{\sigma}(t) u_{0}-\mathbf{i} \int_{0}^{t} S_{\sigma}(t-s)|u(s)|^{2} u(s) d \tau,
$$

and the same for $v$.

Set $w=u-v$ and $\chi_{\epsilon}=1_{\{r \geq \epsilon\}}$.

$$
\left\|w \chi_{\epsilon}\right\|_{L^{2}} \lesssim \int_{0}^{t}\left\|\left(|u|^{2}+|v|^{2}\right) w \chi_{\epsilon}\right\|_{L^{2}} d \tau
$$

Now using the radial Sobolev inequality (2.4), we obtain

$$
|u(r)|^{2}+|v(r)|^{2} \lesssim r^{1^{+}-d}\left(\|u\|_{H^{s}}^{2}+\|v\|_{H^{s}}^{2}\right) \quad r>0 .
$$

Hence

$$
\left\|w \chi_{\epsilon}\right\|_{L^{2}} \lesssim \epsilon^{1^{+}-d} \int_{0}^{t}\left(\|u\|_{H^{s}}^{2}+\|v\|_{H^{s}}^{2}\right)\left\|w \chi_{\epsilon}\right\|_{L^{2}} d \tau .
$$

It follows from the Gronwall that $\left\|w \chi_{\epsilon}\right\|_{L^{2}}=0$. Now, write

$$
\|w\|_{L^{2}} \leq\left\|w \chi_{\epsilon}\right\|_{L^{2}}+\left\|w\left(1-\chi_{\epsilon}\right)\right\|_{L^{2}} \leq\|w\|_{L^{4}}\left\|1-\chi_{\epsilon}\right\|_{L^{4}} .
$$

First, since $u, v \in \Lambda_{s}$, we have that $w^{2} \in L^{2}$ almost surely in the $t$ variable (hence $w \in L^{4}$ almost surely in $t$ ). Second, the Lebesgue dominated convergence theorem shows that $\|1-\chi \epsilon\|_{L^{4}} \rightarrow 0$ as $\epsilon \rightarrow 0$. We obtain $\|w\|_{L^{2}}=0$ almost surely in $t$. Now 
by continuity in $t$, we have that $\|w\|_{L^{2}}=0$ for all $t$. Then the proof of Proposition 6.4 is finished.

As for the continuity, let us present the following result:

Proposition 6.5 For $u_{0}, v_{0} \in \operatorname{supp}(\mu)$, let $u, v$ be the corresponding solutions to (1.1). Let us set $w=u-v$ and $w_{0}=u_{0}-v_{0}$, we have

$$
\|w\|_{L^{2}}^{2} \leq C\left(\left\||u|^{2}\right\|_{L_{t}^{2} H^{\theta}},\left\||v|^{2}\right\|_{L_{t}^{2} H^{\theta}}\right)\left\|w_{0}\right\|_{L^{2}}+\left(\|u\|_{L^{4}}^{2}+\|v\|_{L^{4}}^{2}\right) \epsilon\left(\left\|w_{0}\right\|_{L^{2}}\right),
$$

where $\epsilon\left(\left\|w_{0}\right\|_{L^{2}}\right) \rightarrow 0$ as $\left\|w_{0}\right\|_{L^{2}} \rightarrow 0$.

Proof of Proposition 6.5 Let us take $\theta=\frac{1}{2}+$ and set

$$
\gamma= \begin{cases}\left(-\ln \left\|w_{0}\right\|_{L^{2}}\right)^{\frac{1}{4(2 \theta-d)}} & \text { if } 0<\left\|w_{0}\right\|_{L^{2}}<1 \\ 0 & \text { if } w_{0}=0\end{cases}
$$

Since $\theta<\frac{d}{2}$, we see that $\gamma \rightarrow 0$ as $\left\|w_{0}\right\|_{L^{2}} \rightarrow 0$. So we can consider $\gamma<<1$ and take, as above, the function $\chi_{\gamma}=1_{\{r \geq \gamma\}}$. Using the radial Sobolev inequality (2.4), we have

$$
\left\|w \chi_{\gamma}\right\|_{L^{2}}^{2} \leq\left\|w_{0} \chi_{\gamma}\right\|_{L^{2}}^{2}+C \gamma^{2(2 \theta-d)} \int_{0}^{t}\left(\|u\|_{H^{\theta}}^{2}+\|v\|_{H^{\theta}}^{2}\right)\left\|w \chi_{\gamma}\right\|_{L^{2}}^{2} d \tau .
$$

By the Gronwall inequality we obtain

$$
\begin{aligned}
\left\|w \chi_{\gamma}\right\|_{L^{2}}^{2} & \leq\left\|w_{0} \chi_{\gamma}\right\|_{L^{2}}^{2} e^{\gamma^{2(2 \theta-d)}} C \int_{0}^{t}\left(\|u\|_{H^{\theta}}^{2}+\|v\|_{H^{\theta}}^{2}\right) d \tau \\
& \leq\left\|w_{0} \chi_{\gamma}\right\|_{L^{2}}^{2} e^{\gamma^{4(2 \theta-d)}} e^{C^{2}\left(\int_{0}^{t}\|u\|_{H^{\theta}}^{2}+\|v\|_{H^{\theta}}^{2} d \tau\right)^{2}} \\
& \leq e^{C^{2}\left(\int_{0}^{t}\|u\|_{H^{\theta}}^{2}+\|v\|_{H^{\theta}}^{2} d \tau\right)^{2}}\left\|w_{0}\right\|_{L^{2}} .
\end{aligned}
$$

Therefore

$$
\begin{aligned}
\|w\|_{L^{2}}^{2} & \leq e^{C^{2}\left(\int_{0}^{t}\|u\|_{H^{\theta}}^{2}+\|v\|_{H^{\theta}}^{2} d \tau\right)^{2}}\left\|w_{0}\right\|_{L^{2}}+\left\|w\left(1-\chi_{\gamma}\right)\right\|_{L^{2}}^{2} \\
& \leq e^{C^{2}\left(\int_{0}^{t}\|u\|_{H^{\theta}}^{2}+\|v\|_{H^{\theta}}^{2} d \tau\right)^{2}}\left\|w_{0}\right\|_{L^{2}}+\left\|1-\chi_{\gamma}\right\|_{L^{4}}^{2}\|w\|_{L^{4}}^{2}
\end{aligned}
$$

Since $\left\|1-\chi_{\gamma}\right\|_{L^{4}}^{2} \rightarrow 0$ as $\left\|w_{0}\right\|_{L^{2}} \rightarrow 0$ we arrive at the result of Proposition 6.5.

Notice that Remark 4.5 applies also in the present situation. This completes the proof of Theorem 1.3.

Acknowledgements X.Y. was funded in part by the Jarve Seed Fund and an AMS-Simons travel grant. Both authors are very grateful to the anonymous referees for valuable comments and suggestions. 
Open Access This article is licensed under a Creative Commons Attribution 4.0 International License, which permits use, sharing, adaptation, distribution and reproduction in any medium or format, as long as you give appropriate credit to the original author(s) and the source, provide a link to the Creative Commons licence, and indicate if changes were made. The images or other third party material in this article are included in the article's Creative Commons licence, unless indicated otherwise in a credit line to the material. If material is not included in the article's Creative Commons licence and your intended use is not permitted by statutory regulation or exceeds the permitted use, you will need to obtain permission directly from the copyright holder. To view a copy of this licence, visit http://creativecommons.org/licenses/by/4.0/.

\section{APPENDIX A. Results on probability measures and stochastic pro- cesses}

In this section $(E,\|\|$.$) is a Banach space, C_{b}(E)$ denotes the space of bounded continuous functions $f: E \rightarrow \mathbb{R}$. We present some useful standard results from measure theory.

\section{A.1. Convergence of measures}

Definition A.1 Let $\left(\mu_{n}\right)_{n}$ be a sequence of Borel probability measures and $\mu$ a Borel probability measure on $E$. We say that $\left(\mu_{n}\right)_{n}$ converges weakly to $\mu$ if for all $f \in$ $C_{b}(E)$

$$
\lim _{n \rightarrow \infty} \int_{E} f(x) \mu_{n}(d x)=\int_{E} f(x) \mu(d x)
$$

We write $\mu_{n} \rightarrow \mu$.

Definition A.2 A family $\Lambda$ of Borel probability measures on $E$ is said to be tight if for all $\epsilon>0$ there is a compact set $K_{\epsilon} \subset E$ such that for all $\mu \in \Lambda$

$$
\mu\left(K_{\epsilon}\right) \geq 1-\epsilon .
$$

Theorem A.3 (Portmanteau theorem, see Theorem 11.1.1 in [24]) Let $\left(\mu_{n}\right)_{n}$ be a sequence of probability measures and $\mu$ a probability measure on $E$, the following are equivalent:

(1) $\mu_{n} \rightarrow \mu$,

(2) for all open sets $U$, $\liminf _{n \rightarrow \infty} \mu_{n}(U) \geq \mu(U)$,

(3) for all closed sets $F$, $\lim \sup _{n \rightarrow \infty} \mu_{n}(F) \leq \mu(F)$.

Theorem A.4 (Prokhorov theorem, see Theorem 2.3 in [20]) A set $\Lambda$ of Borel probability measures is relatively compact in $E$ if and only if it is tight.

Theorem A.5 (Skorokhod representation theorem, see Theorem 2.4 in [20]) For any sequence $\left(\mu_{n}\right)_{n}$ of Borel probability measures on E converging weakly to $\mu$, there is a probability space $\left(\Omega_{0}, \mathscr{F}^{0}, \mathbb{P}_{0}\right)$, and random variables $X, X_{1}, \cdots$ on $\left(\Omega_{0}, \mathscr{F}^{0}, \mathbb{P}_{0}\right)$ such that

(1) $\mathscr{L}\left(X_{n}\right)=\mu_{n}$ and $\mathscr{L}(X)=\mu$, 
(2) $\lim _{n \rightarrow \infty} X_{n}=X, \mathbb{P}_{0}$-almost surely.

Here $\mathscr{L}(Y)$ stands for the law of the random variable $Y$.

\section{A.2. Stochastic processes}

Recall that if $\left(X_{t}\right)_{t}$ is an $E$-valued martingale, then since $\|\cdot\|$ is a convex function, we have that $\left(\left\|X_{t}\right\|\right)_{t}$ is a submartingale (by Jensen inequality). Here is a version of the Doob maximal inequality (see Theorem 3.8 in [35]):

Theorem A.6 (Doob maximal inequality) We have that for $p>1$.

$$
\mathbb{E}\left(\sup _{t \in[0, T]}\|X(t)\|\right)^{p} \leq\left(\frac{p}{p-1}\right)^{p} \mathbb{E}\|X(T)\|^{p} .
$$

The following can be found in Chapters 3 and 4 in [47].

Definition A.7 An $N$-dimensional Itô process is a proces $X=\left(X_{t}\right)_{t}$ of the form

$$
X(t)=X(0)+\int_{0}^{t} u(s) d s+\int_{0}^{t} v(s) d B(s)
$$

where $B=\left(B_{i}\right)_{1 \leq i \leq N}$ is an $N$-dimension Brownian motion, $u=\left(u_{i}\right)_{1 \leq i \leq N}$ is an $N$-dimensional stochastic process and $v=\left(v_{i j}\right)_{1 \leq i, j \leq N}$ is a $N \times N$ matrix that both are adapted with respect to $\left(B_{t}\right)_{t}$ and satisfy the following

$$
\mathbb{P}\left(\int_{0}^{t}|u(s)|+|v(s)|^{2} d s<\infty \quad \forall t>0\right)=1 .
$$

A short notation for (6.3) is given by

$$
d X=u d t+v d B
$$

Theorem A.8 ( $N$-dimensional Itô formula) Let $X$ be a $N$-dimensional Itô process as in (6.3). Let $f: \mathbb{R}^{N} \rightarrow \mathbb{R}$ be a $C^{2}$ function, then $f(X)$ is a 1 -dimensional Itô process and satisfy

$$
d f(X)=\nabla f(X) \cdot d X+\frac{1}{2} \sum_{i, j} \partial_{x_{i} x_{j}}^{2} f(X) d X_{i} d X_{j}
$$

with the properties $d B_{i} d B_{j}=\delta_{i j} d t, d t d B_{i}=0$.

Using the properties above, we can remark that in the particular case where $v$ is diagonal (as in this paper), we have

$$
\sum_{i, j} \partial_{x_{i} x_{j}}^{2} f(X) d X_{i} d X_{j}=\sum_{i} \partial_{x_{i}}^{2} f(X) v_{i}^{2} d t .
$$




\section{APPENDIX B. Some important lemmas}

In this section $\left(P_{t}\right)_{t \geq 0}$ be a Feller semi-group (a Markov semi-group satisfying the Feller property) on a Banach space $X, P_{t}^{*}$ is the adjoint operator of $P_{t}$.

Lemma A.9 (Krylov-Bogoliubov argument) If there exists $t_{n} \rightarrow \infty$ and $\mu \in \mathfrak{P}(X)$ such that $\frac{1}{t_{n}} \int_{0}^{t_{n}} P_{t}^{*} \delta_{0} d t \rightarrow \mu$ in $X$, then $P_{t}^{*} \mu=\mu$ for all $t \geq 0$. $\delta_{0}$ is the Dirac measure at 0 .

Proof of Lemma $B$ Note that $P_{r} f \in C_{b}$. We have

$$
\begin{aligned}
\left\langle f, P_{r}^{*} \mu\right\rangle & =\left\langle P_{r} f, \mu\right\rangle=\lim _{t_{n} \rightarrow \infty}\left\langle P_{r} f, \frac{1}{t_{n}} \int_{0}^{t_{n}} P_{t}^{*} \delta_{0} d t\right\rangle=\lim _{t_{n} \rightarrow \infty}\left\langle f, P_{r}^{*} \frac{1}{t_{n}} \int_{0}^{t_{n}} P_{t}^{*} \delta_{0} d t\right\rangle \\
& =\lim _{t_{n} \rightarrow \infty}\left\langle f, \frac{1}{t_{n}} \int_{0}^{t_{n}} P_{t+r}^{*} \delta_{0} d t\right\rangle=\lim _{t_{n} \rightarrow \infty}\left\langle f, \frac{1}{t_{n}} \int_{r}^{t_{n}+r} P_{t}^{*} \delta_{0} d t\right\rangle \\
& =\lim _{t_{n} \rightarrow \infty}\left\langle f, \frac{1}{t_{n}} \int_{0}^{t_{n}} P_{t}^{*} \delta_{0} d t\right\rangle+\left\langle f, \frac{1}{t_{n}} \int_{t_{n}}^{t_{n}+r} P_{t}^{*} \delta_{0} d t\right\rangle-\left\langle f, \frac{1}{t_{n}} \int_{0}^{r} P_{t}^{*} \delta_{0} d t\right\rangle \\
& =\langle f, \mu\rangle
\end{aligned}
$$

where $\lim _{t_{n} \rightarrow \infty}\left\langle f, \frac{1}{t_{n}} \int_{t_{n}}^{t_{n}+r} P_{t}^{*} \delta_{0} d t\right\rangle=0$ and $\lim _{t_{n} \rightarrow \infty}\left\langle f, \frac{1}{t_{n}} \int_{0}^{r} P_{t}^{*} \delta_{0} d t\right\rangle=0$, since $\int_{t_{n}}^{t_{n}+r} P_{t}^{*} \delta_{0} d t$ and $\int_{0}^{r} P_{t}^{*} \delta_{0} d t$ are bounded. Therefore, $P_{r}^{*} \mu=\mu$. Now the proof of Lemma B is finished.

Lemma A.10 Set

$$
v_{n}=\frac{1}{t_{n}} \int_{0}^{t_{n}} P_{t}^{*} \delta_{0} d t .
$$

Assume that $X$ is compactly embedded into a Banach space $X_{0}$ and

$$
\int_{X} f\left(\|u\|_{X}\right) v_{n}(d u) \leq C
$$

for some function $f: \mathbb{R} \rightarrow \mathbb{R}$ such that $\lim _{x \rightarrow \infty} f(x)=\infty$, and $C$ does not depend on $n$. Then there exists $\mu \in \mathfrak{P}(X)$ such that $v_{n} \rightarrow \mu$ weakly on $X_{0}$.

Proof of Lemma A.10 Consider $B_{R}(X)$, by Markov inequality

$$
v_{n}\left(X \backslash B_{R}\right)=v_{n}\left(\|u\|_{X}>R\right) \leq \frac{C}{f(R)},
$$

then $\left(v_{n}\right)$ is tight in $X_{0}$. Now, the Prokhorov theorem (see Theorem A.4) implies the existence of $\mu$ in $\mathfrak{P}\left(X_{0}\right)$.

Now, let us show that $\mu(X)=1$. It suffices to show that

$$
\int_{X_{0}} f\left(\|u\|_{X}\right) \mu(d u) \leq C<\infty
$$


since, with such property, we obtain

$$
\mu\left(B_{R}^{c}\right) \leq \frac{\int_{X_{0}} f\left(\|u\|_{X}\right) \mu(d u)}{f(R)} \rightarrow 0 \quad \text { as } R \rightarrow \infty .
$$

Now, let $\chi_{R}$ be a $C^{\infty}$ function on $[0, \infty)$ such that $\chi_{R}=1$ on $[0, R]$ and $\chi_{R}=0$ on $[R+1, \infty)$.

$$
\int_{X_{0}} f\left(\|u\|_{X}\right) \chi_{R}\left(\|u\|_{X_{0}}\right) v_{n}(d u) \leq \int_{X_{0}} f\left(\|u\|_{X}\right) v_{n}(d u) \leq C .
$$

Since $f\left(\|u\|_{X}\right) \chi_{R}\left(\|u\|_{X_{0}}\right)$ is bounded continuous on $X_{0}$, by Fatou's lemma, we have

$$
\int_{X_{0}} f\left(\|u\|_{X}\right) \mu(d u) \leq C .
$$

The proof of Lemma A.10 is finished.

\section{References}

1. Anton, R.: Cubic nonlinear Schrödinger equation on three dimensional balls with radial data. Comm. Partial Differ. Equ. 33(10-12), 1862-1889 (2008)

2. Ayache, A., Tzvetkov, N.: $L^{p}$ properties for Gaussian random series. Trans. Amer. Math. Soc. 360(8), 4425-4439 (2008)

3. Bedrossian, J., Zelati, M.C., Glatt-Holtz, N.: Invariant measures for passive scalars in the small noise inviscid limit. Commun. Math. Phys. 348(1), 101-127 (2016)

4. Bourgain, J.: Exponential sums and nonlinear Schrödinger equations. Geom. Funct. Anal. 3(2), 157178 (1993)

5. Bourgain, J.: Periodic nonlinear Schrödinger equation and invariant measures. Comm. Math. Phys. 166(1), 1-26 (1994)

6. Bourgain, J.: Invariant measures for the 2D-defocusing nonlinear Schrödinger equation. Comm. Math. Phys. 176(2), 421-445 (1996)

7. Bourgain, J.: On the growth in time of higher Sobolev norms of smooth solutions of Hamiltonian PDE. Int. Math. Res. Notices 1996(6), 277-304 (1996)

8. Bourgain, J.: Refinements of Strichartz' inequality and applications to 2D-NLS with critical nonlinearity. Int. Math. Res, Notices (1998)

9. Bourgain, J., Bulut, A.: Almost sure global well-posedness for the radial nonlinear Schrödinger equation on the unit ball II: the 3d case. J. Eur. Math. Soc. JEMS 16(6), 1289-1325 (2014)

10. Burq, N., Gérard, P., Tzvetkov, N.: Bilinear eigenfunction estimates and the nonlinear Schrödinger equation on surfaces. Invent. Math. 159(1), 187-223 (2005)

11. Burq, N., Thomann, L., Tzvetkov, N.: Remarks on the Gibbs measures for nonlinear dispersive equations. Ann. Fac. Sci. Toulouse Math. 27(3), 527-597 (2018)

12. Carles, R., Faou, E.: Energy cascades for NLS on the torus. Discrete and Continuous Dynamical Systems - A, 32 (2012)

13. Cho, Y., Hajaiej, H., Hwang, G., Ozawa, T.: On the Cauchy problem of fractional Schrödinger equation with Hartree type nonlinearity. Funkcialaj Ekvacioj 56(2), 193-224 (2013)

14. Cho, Y., Ozawa, T.: Sobolev inequalities with symmetry. Commun. Contemp. Math. 11(03), 355-365 (2009)

15. Colliander, J., Keel, M., Staffilani, G., Takaoka, H., Tao, T.: Almost conservation laws and global rough solutions to a nonlinear Schrödinger equation. Math. Res. Lett. 9(5-6), 659-682 (2002)

16. Colliander, J., Keel, M., Staffilani, G., Takaoka, H., Tao, T.: Transfer of energy to high frequencies in the cubic defocusing nonlinear Schrödinger equation. Invent. Math. 181(1), 39-113 (2010) 
17. Constantin, P., Ignatova, M.: Remarks on the fractional laplacian with dirichlet boundary conditions and applications. Int. Math. Res. Notices 2017(6), 1653-1673 (2017)

18. Córdoba, A., Córdoba, D.: A pointwise estimate for fractionary derivatives with applications to partial differential equations. Proc. National Acad. Sci. 100(26), 15316-15317 (2003)

19. Da Prato, G., Debussche, A.: Two-dimensional navier-stokes equations driven by a space-time white noise. J. Funct. Anal. 196(1), 180-210 (2002)

20. Da Prato, G., Zabczyk, J.: Stochastic Equations in Infinite Dimensions. Cambridge University Press (2014)

21. Demirbas, S., Erdogan, M., Tzirakis, N.: In: Lect. Math. (ALM), (ed.) Existence and uniqueness theory for the fractional Schrödinger equation on the torus, volume $34 \mathrm{of} \mathrm{Adv,} \mathrm{pp.} \mathrm{145-162.} \mathrm{Int.} \mathrm{Press,}$ Somerville, MA (2016)

22. Deng, Y., Nahmod, A., Yue, H.: Invariant Gibbs measures and global strong solutions for nonlinear Schröodinger equations in dimension two. arXiv preprint arXiv:1910.08492, (2019)

23. Dinh, V.D.: Well-posedness of nonlinear fractional Schrödinger and wave equations in Sobolev spaces. arXiv preprint arXiv:1609.06181, (2016)

24. Dudley, R.M.: Real Analysis and Probability. Cambridge University Press (2002)

25. Foldes, J., Sy, M.: Invariant measures and global well posedness for SQG equation. arXiv preprint arXiv:2002.09555 (2020)

26. Ginibre, J.: Le problème de Cauchy pour des EDP semi-linéaires périodiques en variables d'espace (d'après Bourgain). Number 237, pages Exp. No. 796, 4, 163-187. (1996). Séminaire Bourbaki, Vol. $1994 / 95$

27. Grande, R.: Continuum limit for discrete NLS with memory effect. arXiv preprint arXiv:1910.05681 (2019)

28. Grande, R.: Space-time fractional nonlinear Schrödinger equation. SIAM J. Math. Anal. 51(5), 41724212 (2019)

29. Guardia, M.: Growth of sobolev norms in the cubic nonlinear Schrödinger equation with a convolution potential. Commun. Math. Phys. 329(1), 405-434 (2014)

30. Guo, B., Huo, Z.: Global well-posedness for the fractional nonlinear Schrödinger equation. Commun. Partial Differ. Equ. 36(2), 247-255 (2010)

31. Hani, Z.: Long-time instability and unbounded sobolev orbits for some periodic nonlinear Schrödinger equations. Arch. Ration. Mech. Anal. 211(3), 929-964 (2014)

32. Hani, Z., Pausader, B., Tzvetkov, N., Visciglia, N.: Modified scattering for the cubic Schrödinger equation on product spaces and applications. In: Forum of mathematics, Pi, volume 3. Cambridge University Press (2015)

33. Hong, Y., Yang, C.: Strong convergence for discrete nonlinear Schrödinger equations in the continuum limit. SIAM J. Math. Anal. 51(2), 1297-1320 (2019)

34. Jonsson, A., Wallin, H.: A Whitney extension theorem in $L_{p}$ and Besov spaces. Annales de l'institut Fourier 28, 139-192 (1978)

35. Karatzas, I., Shreve, S.E.: Brownian Motion and Stochastic Calculus. Springer (1998)

36. Kirkpatrick, K., Lenzmann, E., Staffilani, G.: On the continuum limit for discrete NLS with long-range lattice interactions. Commun. Math. Phys. 317(3), 563-591 (2013)

37. Krengel, U.: Ergodic Theorems, vol. 6. Walter de Gruyter (2011)

38. Kuksin, S.: The Eulerian limit for 2D statistical hydrodynamics. J. Statist. Phys. 115(1-2), 469-492 (2004)

39. Kuksin, S., Shirikyan, A.: Randomly forced CGL equation: stationary measures and the inviscid limit. J. Phys. 37, 3805-3822 (2004)

40. Kuksin, S., Shirikyan, A.: Mathematics of Two-Dimensional Turbulence. Cambridge University Press, Cambridge (2012)

41. Kuksin, S.B.: Oscillations in space-periodic nonlinear Schrödinger equations. Geom. Funct. Anal. GAFA 7(2), 338-363 (1997)

42. Laskin, N.: Fractional quantum mechanics. Phys. Rev. E 62(3), 3135 (2000)

43. Latocca, M.: Construction of high regularity invariant measures for the $2 \mathrm{D}$ and $3 \mathrm{D}$ Euler equations and remarks on the growth of the solutions. arXiv preprint arXiv:2002.11086 (2020)

44. Lebowitz, J.L., Rose, H.A., Speer, E.R.: Statistical mechanics of the nonlinear Schrödinger equation. J. Statist. Phys. 50(3-4), 657-687 (1988)

45. Mattingly, J.C., Pardoux, E.: Invariant measure selection by noise: an example. Discrete and Continuous Dynamical Systems. (2014) 
46. Mingaleev, S.F., Christiansen, P.L., Gaididei, Y.B., Johansson, M., Rasmussen, K.: Models for energy and charge transport and storage in biomolecules. J. Biol. Phys. 25(1), 41-63 (1999)

47. Øksendal, B.: Stochastic Differential Equations. Springer (2003)

48. Shirikyan, A.: Local times for solutions of the complex Ginzburg-Landau equation and the inviscid limit. J. Math. Anal. Appl. 384(1), 130-137 (2011)

49. Staffilani, G.: On the growth of high Sobolev norms of solutions for KdV and Schrödinger equations. Duke Math. J. 86(1), 109-142 (1997)

50. Sun, C., Tzvetkov, N.: Gibbs measure dynamics for the fractional nls. SIAM J. Math. Anal. 52(5), 4638-4704 (2020)

51. Sy, M.: Invariant measure and long time behavior of regular solutions of the Benjamin-Ono equation. Anal. PDE 11(8), 1841-1879 (2018)

52. Sy, M.: Almost sure global well-posedness for the energy supercritical Schrödinger equations. arXiv preprint arXiv:1905.03866 (2019)

53. Sy, M.: Invariant measure and large time dynamics of the cubic Klein-Gordon equation in 3D. Stochast. Partial Differ. Equ. Anal. Comput. 7(3), 379-416 (2019)

54. Sy, M., Yu, X.: Almost sure global well-posedness for the energy supercritical NLS on the unit ball of $\mathbb{R}^{3}$. arXiv preprint arXiv:2007.00766 (2020)

55. Sy, M., Yu, X.: Global well-posedness for the cubic fractional NLS on the unit disk. arXiv preprint arXiv:2011.05517, (2020)

56. Tzvetkov, N.: Invariant measures for the nonlinear Schrödinger equation on the disc. Dyn. Partial Differ. Equ. 3(2), 111-160 (2006)

57. Tzvetkov, N.: Invariant measures for the defocusing nonlinear Schrödinger equation. Ann. Inst. Fourier (Grenoble) 58(7), 2543-2604 (2008)

Publisher's Note Springer Nature remains neutral with regard to jurisdictional claims in published maps and institutional affiliations. 